\title{
Elp1 facilitates RAD51-mediated homologous recombination repair via translational regulation
}

\author{
Wei-Ting Chen ${ }^{1}$, Huan-Yi Tseng ${ }^{1}$, Chung-Lin Jiang ${ }^{1}$, Chih-Ying Lee ${ }^{2}$, Peter Chi ${ }^{2,3}$, Liuh-Yow Chen ${ }^{4}$, Kai-Yin Lo ${ }^{5}$, \\ I-Ching Wang ${ }^{6}$ and Fu-Jung Lin ${ }^{1,7^{*}}$ (D)
}

\begin{abstract}
Background: RAD51-dependent homologous recombination (HR) is one of the most important pathways for repairing DNA double-strand breaks (DSBs), and its regulation is crucial to maintain genome integrity. Elp1 gene encodes IKAP/ELP1, a core subunit of the Elongator complex, which has been implicated in translational regulation. However, how ELP1 contributes to genome maintenance is unclear.
\end{abstract}

Methods: To investigate the function of Elp1, Elp1-deficient mouse embryonic fibroblasts (MEFs) were generated. Metaphase chromosome spreading, immunofluorescence, and comet assays were used to access chromosome abnormalities and DSB formation. Functional roles of Elp1 in MEFs were evaluated by cell viability, colony forming capacity, and apoptosis assays. HR-dependent DNA repair was assessed by reporter assay, immunofluorescence, and western blot. Polysome profiling was used to evaluate translational efficiency. Differentially expressed proteins and signaling pathways were identified using a label-free liquid chromatography-tandem mass spectrometry (LC-MS/MS) proteomics approach.

Results: Here, we report that Elp1 depletion enhanced genomic instability, manifested as chromosome breakage and genotoxic stress-induced genomic DNA fragmentation upon ionizing radiation (IR) exposure. Elp1-deficient cells were hypersensitive to DNA damage and exhibited impaired cell proliferation and defective HR repair. Moreover, Elp1 depletion reduced the formation of IR-induced RAD51 foci and decreased RAD51 protein levels. Polysome profiling analysis revealed that ELP1 regulated RAD51 expression by promoting its translation in response to DNA damage. Notably, the requirement for ELP1 in DSB repair could be partially rescued in Elp1-deficient cells by reintroducing RAD51, suggesting that Elp1-mediated HR-directed repair of DSBs is RAD51-dependent. Finally, using proteome analyses, we identified several proteins involved in cancer pathways and DNA damage responses as being differentially expressed upon Elp1 depletion.

Conclusions: Our study uncovered a molecular mechanism underlying Elp1-mediated regulation of HR activity and provides a novel link between translational regulation and genome stability.

Keywords: Elp1, RAD51, Homologous recombination, DNA damage, Translational regulation

*Correspondence: fujlin@ntu.edu.tw

1 Department of Biochemical Science and Technology, National Taiwan University, No.1, Sec.4, Roosevelt Rd., Taipei 10617, Taiwan

Full list of author information is available at the end of the article

\section{Background}

Genomic instability is a common feature of most cancer cells and it is considered an additive impact of DNA damage, impaired DNA repair, and a failure to block cell cycle progression before the damaged DNA has been passed original author(s) and the source, provide a link to the Creative Commons licence, and indicate if changes were made. The images or other third party material in this article are included in the article's Creative Commons licence, unless indicated otherwise in a credit line to the material. If material is not included in the article's Creative Commons licence and your intended use is not permitted by statutory regulation or exceeds the permitted use, you will need to obtain permission directly from the copyright holder. To view a copy of this licence, visit http://creativecommons.org/licenses/by/4.0/. The Creative Commons Public Domain Dedication waiver (http://creativeco mmons.org/publicdomain/zero/1.0/) applies to the data made available in this article, unless otherwise stated in a credit line to the data. 
on to daughter cells. Maintenance of genomic integrity and the high fidelity of DNA damage repair mechanisms are fundamental processes for ensuring proper cell growth, development, and survival.

DNA double-strand breaks (DSBs), the most deleterious type of DNA damage, can alter genomic stability and lead to cell death. In mammalian cells, DSBs are induced by exposure to various external hazards, such as $\mathrm{UV}$, ionizing radiation (IR), and interstrand crosslinking agents. DSBs also arise normally during DNA replication and other cellular process, such as $\mathrm{V}(\mathrm{D}) \mathrm{J}$ recombination, meiotic DNA crossover formation, and upon attack by endogenous reactive oxygen species [1]. To prevent accumulation of DNA lesions, a DNA damage response (DDR) can be activated, or genes involved in promoting apoptosis or senescence are induced. The two predominant mechanisms for repairing DSBs are homologous recombination (HR) and non-homologous end-joining (NHEJ) [2, 3]. In general, upon detecting DNA damage, a group of DDR factors is rapidly recruited to the damaged sites, which together activate cell cycle checkpoints and promote DNA repair pathways. Defects in the DNA damage repair response can result in cell death and a number of diseases.

HR is critical for error-free repair of broken chromosomes, such as collapsed replication forks and DNA interstrand crosslinks. It requires the presence of a homologous DNA region as a template for accurate repair. An early step of HR repair is DNA end resection, which generates a single-stranded DNA (ssDNA) tail to initiate the recombination process. Upon recruitment of HR components, the recombinase RAD51 forms a nucleoprotein filament and catalyzes DNA strand exchange with an undamaged sister chromatid, with this latter serving as the DNA template for high-fidelity repairs [4-6]. RAD51-containing core protein complexes in conjunction with context-specific factors are needed for a number of interrelated HR pathways. As RAD51 is the major strand transfer protein in HR-mediated DNA repair, a reduction in the formation of RAD51 foci at DSBs implies impaired recruitment of repair proteins and diminished HR-dependent DSB repair.

Elongator is a highly conserved multisubunit complex that consists of two copies of each of its six subunits (Elp1-6). Every subunit is functionally essential in vivo as deletion of any of them leads to the loss of complex integrity and generates almost identical pleiotropic phenotypes [7]. ELP1 (also known as IKAP and encoded by Elp1/Ikbkap gene) is a scaffold protein of Elongator. ELP1 has been shown to play a key role not only in transcription, but also in translation [7, 8]. Mutations in the human IKBKAP/ELP1 gene, resulting in severely reduced IKAP/ELP1 protein levels in the nervous system, cause the autosomal-recessive neurodevelopmental disorder-familial dysautonomia (FD) $[9,10]$. Mice lacking Elp1 exhibit developmental delay and early embryonic lethality [11]. Peripheral neural-specific deletion of Elp1 in mice results in increased apoptosis of neural progenitors and peripheral neurons [12]. Recently, germline loss-of-function $I K B K A P / E L P 1$ variants were identified in patients with pediatric Sonic Hedgehog medulloblastomas, highlighting the role of Elongator and its subunits in tumor development [13]. Apart from its cell autonomous role in neurogenesis, several lines of evidence support a role for ELP1 in suppressing genomic instability. Elp1-deficient mouse spermatocytes exhibit defects in DSB repair [14]. In yeast, Elongator mutants are hypersensitive to the DNA damage reagents methyl methanesulfonate (MMS) and hydroxyurea [15]. Although the phenotypes arising from Elp1 deficiency in mice and yeasts often reflect impaired genome stability, a role for ELP1 in maintaining genomic stability has not yet been systematically established.

Here, we show definitively that Elp1 plays a role in genome maintenance. Deletion of Elp1 results in genotoxic agent-induced chromosomal instability. Elp1-deficient mouse embryonic fibroblasts (MEFs) were deficient in HR repair and hypersensitive to IR and chemicals that induce DSBs, even culminating in cell death. Elp1 facilitated HR-directed DSB repair, at least in part, by enhancing Rad51 translational efficiency and by promoting its expression. Finally, by means of a mass spectrometry (MS)-based proteomics approach and bioinformatics tools, we show that differentially expressed proteins in Elp1-deficient MEFs were most significantly enriched in pathways related to cancer, apoptosis, and DNA repair. Overall, our study uncovers a crucial role for Elp1 in genome integrity.

\section{Materials and methods}

Generation of Elp $1^{\text {flox/flox }}$ MEFs and cell culture

Elp1 $1^{\text {flox/flox }}$ mice [14] were provided by Yi Zhang (Harvard Medical School, MA, USA). Elp $1^{\text {flox/flox }}$ MEFs were isolated from embryonic day (E) $13.5 E$ Elp $^{\text {flox/flox }}$ embryos according to standard protocols. In brief, after removing the head and innards of embryos, the remaining tissue was minced and then incubated overnight with $0.25 \%$ trypsin/EDTA at $4{ }^{\circ} \mathrm{C}$. The supernatant was extracted and supplemented with complete medium. After cell dissociation, the cellular suspension was transferred to $10 \mathrm{~cm}$ plates. Once the cells had grown to confluency, they were collected and stored in liquid nitrogen (passage $1 \mathrm{MEFs}$ ). These primary MEFs were cultured in Dulbecco's Modified Eagle's Medium (DMEM) containing $10 \%$ fetal bovine serum (HyClone Laboratories, Logan, UT, USA), $100 \mathrm{U} / \mathrm{mL}$ penicillin/streptomycin (HyClone 
Laboratories), $0.1 \mathrm{mM}$ non-essential amino acids (HyClone Laboratories), and $50 \mu \mathrm{M} \beta$-mercaptoethanol (Sigma-Aldrich, St. Louis, MO, USA). To immortalize primary MEFs, early passage (P2) cells were immortalized via retroviral infections with pBabeSV40LT (Addgene \#13970) and then selected with G418 (Life Technologies, Carlsbad, CA, USA) for 7 days. Immortalized MEFs were cultured in the same DMEM medium but without addition of $\beta$-mercaptoethanol. Human osteosarcoma U2OS cells containing the DR-GFP or EJ5-GFP reporter system were provided by Jeremy M. Stark (City of Hope Medical Center, CA, USA) [16], and they were cultured in DMEM containing $10 \%$ fetal bovine serum, penicillin-streptomycin and sodium pyruvate, and maintained at $37{ }^{\circ} \mathrm{C}$ in an incubator at $5 \% \mathrm{CO}_{2}, 95 \%$ humidity.

\section{Knockout or knockdown of Elp1}

To knock out Elp1 from Elp $1^{\text {flox/flox }}$ MEFs, we used control (pLKO.1) and Cre-expressing lentiviruses. The Creexpressing lentiviral vectors were provided by I-Ching Wang (National Tsing Hua University, Taiwan). To knock down ELP1 from U2OS cells, we used control (shCtrl, pLKO_TRC025), and two independent ELP1 shRNAs, shELP1-73 (clone ID: TRCN0000037873, target sequence: GCTGTGCTCTTGCTGTTAGAA), and shELP1-69 (clone ID: TRCN0000037869, target sequence: GCGTCAAATATCACGTCATTT) lentiviruses. All viruses were obtained from the RNAi Core of Academia Sinica, Taiwan. Cells were incubated with viral supernatant (M.O.I. $=4)$ supplemented with $6 \mu \mathrm{g} / \mathrm{mL}$ polybrene (Sigma-Aldrich) for $24 \mathrm{~h}$, followed by puromycin-based selection (InvivoGen, San Diego, CA, USA).

\section{Transfections and DSB reporter assays}

Two days after shRNA virus infection, I-SceI expression plasmids containing a hemagglutinin (HA) tag (pCBASceI, Addgene \#26477) or pcDNA3.1 control plasmids were transfected into U2OS cells using Lipofectamine 3000 (Thermo Fisher Scientific, Waltham, MA, USA) according to the manufacturer's protocol. One day after transfection, cells were harvested and analyzed for the percentage of GFP-positive cells by means of flow cytometry (FACSCanto II, BD Biosciences, San Jose, CA, USA). Data were analyzed in FlowJo software (version 9.4.2; Treestar, Ashland, OR, USA).

\section{Immunofluorescence staining}

Cells grown on coverslips were fixed in $4 \%$ paraformaldehyde for $20 \mathrm{~min}$, followed by permeabilization with $0.2 \%$ Triton X-100 in PBS for 10 min. After blocking, coverslips were incubated overnight with anti- $\gamma \mathrm{H} 2 \mathrm{AX}$ (ab22551, 1:1000, Abcam, Cambridge, UK), anti-ELP1 (ab62498, 1:500, Abcam), anti-RAD51 (PC130, 1:500, Merck
Millipore, Burlington, MA, USA) or RPA70 (GTX108749, 1:400, GeneTex, Irvine, CA, USA) antibodies at $4{ }^{\circ} \mathrm{C}$. Following several washes with $0.1 \%$ Triton X-100/PBS buffer, coverslips were incubated with Alexa Fluor 488-conjugated anti-mouse or Alexa Fluor 594-conjugated antirabbit secondary antibodies (\#A11001 or \#A11012, 1:500, Invitrogen, Waltham, MA, USA) for $1 \mathrm{~h}$. DAPI was used to stain nuclei. Fluorescence mounting medium (Agilent Dako, Santa Clara, CA, USA) was used to mount coverslips. Images were taken with a Leica TCS SP5 II Confocal microscope (Leica Microsystems, Wetzlar, Germany) or Zeiss AxioImager M2 microscope (Zeiss, Oberkochen, Germany). Images were analyzed using Axiovision or Leica Application Suite X software.

\section{Metaphase chromosome spread}

Cells were incubated with $0.1 \mu \mathrm{g} / \mathrm{mL}$ colcemid (Thermo Fisher Scientific) for $12 \mathrm{~h}$ to block cell cycle progression at metaphase. Following trypsinization, cells were washed twice with ice-cold PBS, and pelleted by centrifugation. The cell pellet was treated in hypotonic solution ( $75 \mathrm{mM} \mathrm{KCl}$ ) at $37{ }^{\circ} \mathrm{C}$ for $20 \mathrm{~min}$ and fixed in a fixative with methanol and acetic acid $(2: 1, \mathrm{v} / \mathrm{v})$ for at least $4 \mathrm{~h}$ at $-20{ }^{\circ} \mathrm{C}$. Cells were re-suspended in freshly prepared fixative, and the fixed cells were dropped on clean glass slides and air-dried. The slides were stained with DAPI to visualize chromosomes. Images were taken with a Leica TCS SP5 II Confocal microscope (Leica Microsystems). Standard criteria were used for scoring metaphase cells. At least 250 cells at metaphase were analyzed per sample.

\section{Analysis of micronuclei and nuclear buds}

Cells were infected with control or Cre-expressing viruses at D0 and harvested at D5. Cells containing micronuclei and nuclear buds were identified by DAPI staining. Micronuclei were scored when they did not attach to the main nucleus and had no connection to it [17]. Nuclear buds were counted only when they touched the main nucleus by a nucleoplasmic connection that was thinner than the diameter of the bud [18]. Microscopic images were obtained using a Zeiss AxioImager M2 microscope (Zeiss).

\section{Comet assay}

Cells were seeded in 12-well plates and infected with control or Cre-expressing viruses for 4 days. Once cells reached $70 \%$ confluency at day 4 , a total of $0.75 \mu \mathrm{g}$ plasmid DNA (hRAD51-expressing or control vectors) was transfected into MEFs using Lipofectamine 3000 (Thermo Fisher Scientific). One day after transfection, cells were washed twice with PBS and irradiated with a 4 Gy IR. At day 6, a comet assay using CometSlides ${ }^{\mathrm{TM}}$ (Trevigen, Gaitherburg, MD, USA) under neutral 
conditions was performed according to the manufacturer's protocol. In brief, cells were mixed with $1 \%$ low-melting temperature agarose and spread onto CometSlides ${ }^{\mathrm{TM}}$. Slides were immersed in lysis buffer containing $1 \%$ Triton $\mathrm{X}-100$ for $40 \mathrm{~min}$ at $4{ }^{\circ} \mathrm{C}$, and then subjected to electrophoresis at $30 \mathrm{~V}$ for $35 \mathrm{~min}$ at $4{ }^{\circ} \mathrm{C}$, followed by staining with EtBr solution (Sigma-Aldrich). Images encompassing $>50$ randomly selected cells per sample were taken using a Zeiss AxioImager M2 microscope (Zeiss). The tail moment was computed as the percentage of DNA in the comet tail multiplied by the tail length in individual nuclei using the TriTek CometScore Freeware 1.6.1.13 (TriTek Technologies, Inc., Wilmington, DE, USA).

\section{Cell functional assays}

For detection of apoptotic cells, cells were infected with control or Cre-expressing viruses for 5 days and exposed to etoposide (ETO) (Sigma-Aldrich) or irradiation as indicated. Apoptotic cells were stained and quantified using a FITC Annexin V Apoptosis Detection Kit with 7-amino-actinomycin-D (7-AAD; BioLegend Biotechnology, San Diego, CA, USA). For the cell viability assay, $1 \times 10^{4}$ cells were seeded in 6-well plates one day before being infected with control or $\mathrm{Cre}$-expressing lentivirus $($ M.O.I. $=4)$. Five days after virus infection (day 5$)$, cells were exposed to IR (4 Gy) (IBL 637 Cesium-137 $\gamma$-ray machine, Cisbio International, Saclay, France). Cell numbers were counted at days 3, 4, 6, and 9. For clonogenic experiments, 3000 cells were seeded in 6-well plates. Cells were infected with lentivirus, treated with puromycin, and exposed to irradiation as indicated. Cells were allowed to grow and on day 10 were stained with $0.1 \%$ crystal violet. Quantitative changes in clonogenicity were determined by extracting the colonies with acetic acid and measuring the absorbance of the extracted dye at $592 \mathrm{~nm}$. For cell cycle analysis, cells were harvested and fixed in $70 \%$ ethanol at $4{ }^{\circ} \mathrm{C}$ for $24 \mathrm{~h}$. Prior to analysis, the fixed cells were washed with PBS and stained in PBS containing propidium iodide $(10 \mu \mathrm{g} / \mathrm{mL})$ (BioLegend Biotechnology), RNase $(10 \mathrm{U} / \mathrm{mL})$, and triton X-100 (0.1\% $\mathrm{v} / \mathrm{v}$ ). Analysis was performed using a FACSCalibur flow cytometer (BD Biosciences).

\section{Quantitative real-time-PCR assay}

Total RNAs were extracted from cells using QIAzol (Qiagen, Hilden, Germany). Complementary DNAs were generated by SuperScriptIV reverse transcriptase (Thermo Fisher Scientific). Quantitative RT-PCR analyses were performed with a QuantStudio 3 Real-Time PCR System (Applied Biosystems, Waltham, MA, USA). Data are presented as fold-change in gene expression, normalized to $18 \mathrm{~S}$ ribosomal RNA. The primer sequences are listed in Additional file 1: Table S1.

\section{Western blot analysis}

Cells were lysed with $1 \times$ RIPA buffer (Thermo Fisher Scientific) supplemented with protease and phosphatase inhibitor cocktail (Roche Applied Science, Upper Bavaria, Germany) for 30 min on ice. After centrifugation for $10 \mathrm{~min}$, the supernatant was collected and the protein content of the samples was analyzed by means of BCA Protein Assay (T-Pro Biotechnology, Taipei, Taiwan). Proteins were loaded onto SDS-polyacrylamide gels and blotted onto PVDF membranes (GE Healthcare Life Science, Marlborough, MA, USA). Western blots were performed using primary antibodies directed against ELP1 (ab62498, 1:1500, Abcam), RAD51 (PC130, 1:2000, Merck Millipore), HA (GTX115044, 1:200, GeneTex), p53 (\#2524, 1:1000, Cell Signaling Technology, Danvers, MA, USA), phospho-p53 (\#9284, 1:1000, Cell Signaling Technology), GAPDH (GTX100118, 1:5000, GeneTex) and $\beta$-actin (GTX109639, 1:10000, GeneTex). Horseradish peroxidase-linked anti-mouse or anti-rabbit conjugates were used as secondary antibodies (1:5000, Jackson ImmunoResearch, West Grove, PA, USA). Visualization was carried out by enhanced chemiluminescence development (T-Pro Biotechnology).

\section{Polysome profiling analysis}

At least $3 \times 10^{6}$ cells were lysed with polysome lysis buffer (200 mM NaCl, 15 mM MgCl, 15 mM Tris- $\mathrm{HCl}$ pH 7.3, $1 \%$ Triton X-100, $0.1 \mathrm{mg} / \mathrm{mL}$ cycloheximide) on ice for $10 \mathrm{~min}$ and centrifuged at $13,300 \mathrm{rpm}$ for $10 \mathrm{~min}$ at $4{ }^{\circ} \mathrm{C}$. The supernatant containing $300 \mu \mathrm{g}$ RNA was loaded onto the top of a $10-50 \%$ sucrose gradient and centrifuged at 40,000 rpm for $2 \mathrm{~h}$ at $4{ }^{\circ} \mathrm{C}$ using a Beckman L8-60M Ultracentrifuge (Beckman Coulter, Brea, CA, USA). By continuous measurement of absorbance at $254 \mathrm{~nm}$, the gradient was collected into 13 fractions ( $1 \mathrm{~mL} /$ fraction) using a gradient fractionator (BioComp Instruments, Fredericton, Canada). RNAs in each fraction were isolated and analyzed.

\section{Protein preparation and liquid chromatography-tandem mass spectrometry (LC-MS/MS) analysis}

Cells were lysed and proteins were precipitated with acetone. Following BCA-based quantification of protein concentration (T-Pro Biotechnology), extracted protein was reduced in $5 \mathrm{mM}$ dithiothreitol (DTT) and alkylated in $10 \mathrm{mM}$ iodoacetamide. It was further digested with trypsin at $29^{\circ} \mathrm{C}$ for $16 \mathrm{~h}$, and the reaction was stopped by adding $10 \%$ trifluoroacetic acid (TFA) to a final concentration of $0.5 \%$. Digested peptides were desalted by using $\mathrm{C} 18$ before being analyzed by $\mathrm{LC}-$ MS/MS using an Orbitrap Fusion Lumos Tribrid quadrupole-ion trap-Orbitrap mass spectrometer (Thermo Fisher Scientific) coupled to a UHPLC system (UltiMate 
3000, Thermo Fisher Scientific). Peptide mixtures were loaded onto the analytical column and separated by reversed-phase chromatography using a $25-\mathrm{cm}$ column with an inner diameter of $75 \mu \mathrm{m}$, packed with $2 \mu \mathrm{m} \mathrm{C}_{18}$ particles (Acclaim PepMap RSLC; Thermo Fisher Scientific). The protein solution was run with a gradient concentration of acetonitrile ranging from 2 to $40 \%$ in $0.1 \%$ formic acid in acetonitrile at a flow rate of $300 \mathrm{~nL} /$ min for $90 \mathrm{~min}$. Positive ions were generated by electrospray and the Orbitrap was operated in data-dependent acquisition (DDA) mode. A survey scan 350-1750 m/z was acquired in the Orbitrap (resolution $=120,000$ at $200 \mathrm{~m} / z$, with the AGC target of 500,000 ions). Highenergy collision activated dissociation (HCD)-MS/ MS (resolution of 15,000) was used to fragment multiply-charged ions within a $1.4 \mathrm{Da}$ isolation window at normalized collision energy of 32. An AGC target of 50,000 was set for MS/MS analysis with previously selected ions dynamically excluded for 180 s. Samples were analyzed in duplicate. All MS/MS spectral data were searched against the mouse database from the UniProt website (http://www.uniprot.org) using MASCOT 2.3 (Matrix Science, London, UK) via the Proteome Discoverer (PD) package (version 2.2, Thermo Fisher Scientific). The following parameter settings were used: trypsin cleavage; two missed cleavage sites; modification allowed for cysteine carbamidomethylation and methionine oxidation. Peptide mass tolerance was set to $\pm 10 \mathrm{ppm}$, and fragment MS/MS tolerance was set to $0.02 \mathrm{Da}$, and a $1 \%$ false discovery rate (FDR) was accepted. For relative protein quantification across different samples, each protein group is represented by a single master protein and the raw abundance of each protein was normalized by total abundance. At least two independent samples per condition were analyzed.

\section{Ingenuity pathway analysis}

Differentially expressed proteins were analyzed by Ingenuity pathway analysis (IPA, www.ingenuity.com) (Qiagen, Redwood City, CA, USA) to identify canonical pathways, associated diseases, biological functions, and upstream regulators.

\section{Statistical analysis}

Results are presented as mean \pm standard error of the mean (SEM). All datasets were tested for normality for Student's $t$-test, and if the normality test failed, a MannWhitney rank-sum test was used instead. Statistical analyses were performed using SigmaPlot 12.5 or Microsoft Excel. $p$ values $<0.05$ were considered significant. * $p<0.05$, ${ }^{* *} p<0.01$, and ${ }^{* * *} p<0.005$.

\section{Results}

Elp1 prevents genomic instability

To generate Elp1 conditional knockout cells, we isolated Elp1 $1^{\text {flox/flox }}$ MEFs from mice harboring an Elp1 conditional knockout allele in which two loxP sites were inserted on either side of exon 4 (Additional file 1: Fig. S1). Cre recombinase was then introduced by lentiviral infection to generate Elp1-deficient (KO) MEFs. Puromycin was added on the next day to enrich for infected cells. Five days after Cre introduction, a low-dose IR treatment (4 Gy) was applied to induce DSBs (Fig. 1A). We found that Elp1 transcripts were significantly reduced by that time-point, as evidenced by qRT-PCR analysis (Fig. 1B). Immunofluorescence staining also revealed that ELP1 is predominantly expressed in the cytoplasm of control (Ctrl) MEFs, whereas ELP1 signal was marked diminished in KO MEFs (Fig. 1C). Chromosomal instability is usually characterized by a high frequency of nuclear buds (NBUDs) and micronuclei (MNi). We examined the effect of Elp1 knockout on these biomarkers of chromosomal damage, which revealed higher incidences of NBUD and MNi formation in KO MEFs relative to Ctrl cells (Fig. 1D). To directly assess if Elp1 deletion increases genomic instability, we performed cytogenetic analysis of chromosomes in MEFs following low-dose IR treatment. Elp1 transcripts were significantly reduced at day 3 (Additional file 1: Fig. S2). We observed an elevated frequency of chromosomal abnormalities, including at least two-fold increases in chromosomal breakage, in $\mathrm{KO}$ MEFs compared to Ctrl cells (Fig. 1E). These results suggest an increased level of genomic instability in $\mathrm{KO}$ cells.

\section{KO MEFs are hypersensitive to IR-induced DNA damage}

Defects in genome maintenance lead to an accumulation of unrepaired DNA lesions and the activation of the DDR [19]. To establish if Elp1 deficiency promotes DDR activation, we assayed for formation of phosphorylated histone variant $\mathrm{H} 2 \mathrm{AX}(\gamma \mathrm{H} 2 \mathrm{AX})$ foci in $\mathrm{KO}$ cells with or without IR (4 Gy), as $\gamma \mathrm{H} 2 \mathrm{AX}$ is indicative of unrepaired or irreparably damaged DNA [20]. $\gamma \mathrm{H} 2 \mathrm{AX}$ strongly promotes the recruitment of DSB repair proteins, with the process of repair usually being completed within $24 \mathrm{~h}$ after DSB induction [21]. We found that the percentage of cells with $\gamma \mathrm{H} 2 \mathrm{AX}$-positive foci was significantly higher in $\mathrm{KO}$ cells compared to Ctrl cells at 4 and $24 \mathrm{~h}$ following irradiation (Fig. 2A, B), indicating that $\mathrm{KO}$ cells are less effective at repairing DSBs. To directly determine the effect of Elp1 knockout on DNA damage, we performed a comet assay. In the absence of IR treatment, amounts of DSBs were comparable between Ctrl and KO cells (Fig. 2C, D). However, upon IR treatment, we observed a significant increase in DNA migration, manifested as comet tails, for $\mathrm{KO}$ 
A

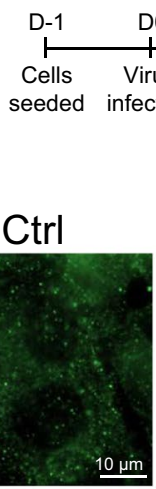

$\mathrm{KO}$

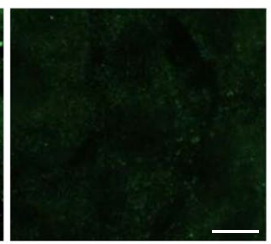

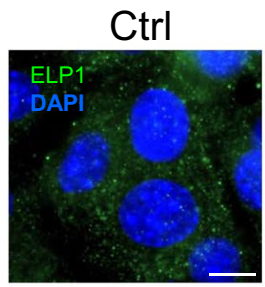

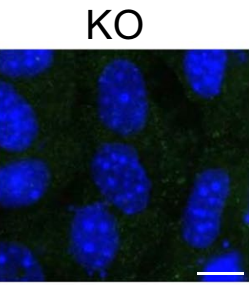

KO
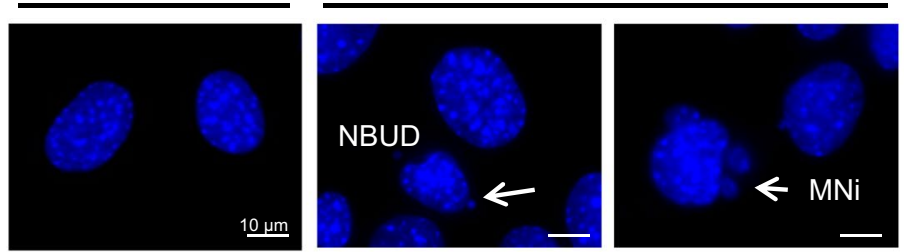

B
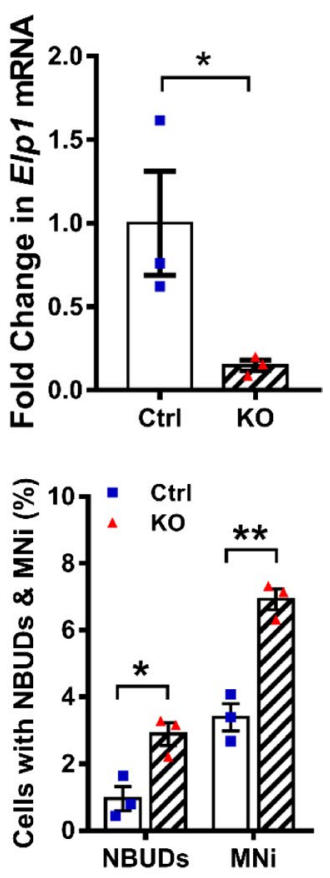

$\mathbf{E}$
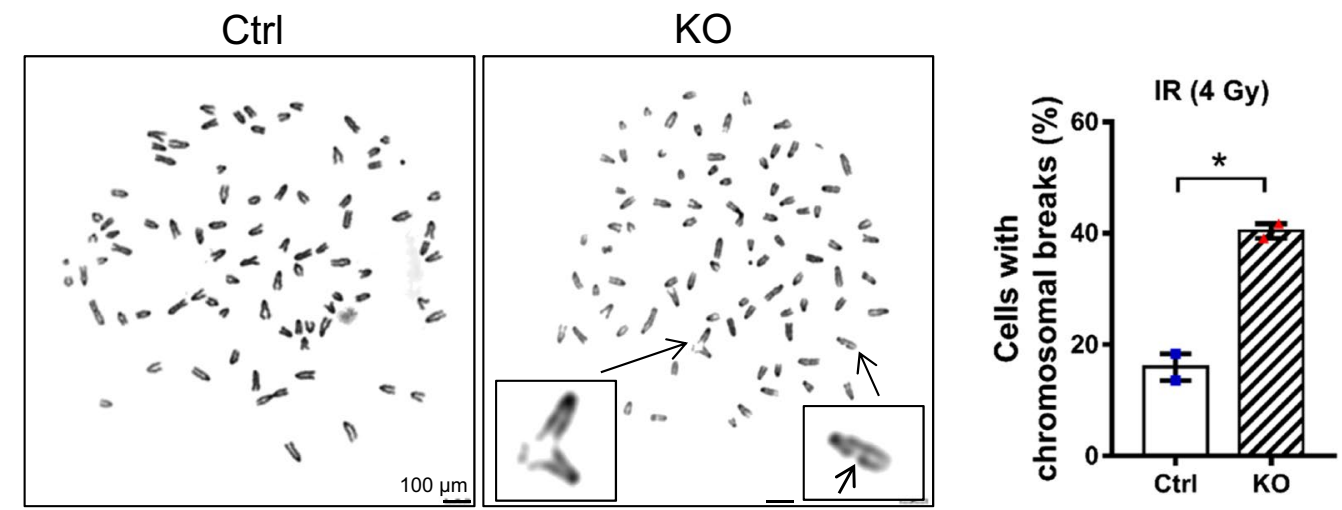

Fig. 1 Elp 1 knockout results in chromosomal instability. A Schematic of our experimental design. Infected cells were irradiated with a single acute dose of 4 Gy IR at day (D) 5 unless otherwise described. B qRT-PCR analysis of the Elp 1 mRNA expression by D5 MEFs. C Immunofluorescence for ELP1 (green) in D5 MEFs. Nuclei were counterstained with DAPI (blue). Scale bars, $10 \mu \mathrm{m}$. D Representative images of nuclear buds (NBUDs), and micronuclei (MNi) in the Ctrl and KO MEFs. Scale bars, $10 \mu \mathrm{m}$. Statistical results were obtained from analyzing at least 100 cells in each group. Data represent the average of three independent experiments. $\mathbf{E}$ Ctrl and KO MEFs were exposed to 4 Gy of IR at D2, and chromosome breaks were counted in a minimum of 100 metaphase cells in each group at D3. Representative images of chromosomal spreads for Ctrl and KO MEFs are shown in the left panel, and quantification of the percentage of cells displaying chromosome breaks is presented in the right panel. Arrows indicate DNA breaks. Data represent the average of two independent experiments. Results are presented as mean \pm SEM. Scale bars, $100 \mu \mathrm{m} .{ }^{*} p<0.05 ;{ }^{* *} p<0.01$ compared to Ctrl cells using Student's t-test

MEFs compared to Ctrl cells (Fig. 2C), which was confirmed by statistical analyses of the percentage of tail DNA and the extent of tail moment (Fig. 2D). These results indicate that unrepaired DNA damage following IR was significantly higher in $\mathrm{KO}$ cells relative to Ctrl MEFs.

\section{Elp1 knockout enhances levels of DNA damage} in the presence of genotoxic stress

To further demonstrate DDR activation upon Elp1 deletion, we treated MEFs with ETO, a compound that targets DNA topoisomerase II and that promotes DSB production. As observed for IR treatment, we observed 
significantly higher numbers of $\gamma \mathrm{H} 2 \mathrm{AX}$ foci in KO MEFs compared to Ctrl MEFs at 16 and $24 \mathrm{~h}$ after drug removal (Fig. 2E, F), demonstrating that Elp1 knockout renders MEFs more susceptible to genotoxic agent-induced DSB accumulation. Numbers of $\gamma \mathrm{H} 2 \mathrm{AX}$ foci were comparable in Ctrl and KO MEFs at the earlier time-point of $2 \mathrm{~h}$ (Fig. 2E, F), evidencing that ETO treatment had induced similar levels of DNA damage. These results indicate that KO MEFs are hypersensitive to genotoxic stress relative to Ctrl MEFs and further reveal that KO MEFs are less effective in DNA damage repair rather than being more susceptible to DNA damage.

\section{Elp1 deficiency and DNA damage together reduce cell division and induce apoptosis}

To examine the functional consequences of defective DSB repair in KO cells, we assayed cell viability in the presence or absence of IR. In the absence of IR treatment, we observed a significant decrease in cell division six or nine days after induction of Elp1 deletion relative to Ctrl cells (Fig. 3A). Moreover, combinatorial Elp1 knockout and radiation treatment was additive in terms of reducing cell division (Fig. 3A), revealing that Elp1 knockout alone or in combination with IR treatment was sufficient to compromise cell division. We further analyzed the cell cycle profiles of Ctrl and KO cells using flow cytometry and propidium iodide staining. In the absence of IR treatment, knockout of Elp1 significantly increased the proportion of cells in the G1 phase and reduced the proportion of cells in the $\mathrm{S}$ phase of the cell cycle (Additional file 1: Fig. S3), suggesting G1 cell cycle arrest in KO cells. Next, we employed a colony formation assay to further confirm these results. As shown in Fig. 3B, the colonyforming capacity of KO MEFs was significantly impaired when compared to Ctrl MEFs. As anticipated, combining Elp1 knockout with IR further reduced cell clonogenicity (Fig. 3B).

To characterize a role for Elp1 in DNA damage repair, we analyzed apoptotic rates of Ctrl and KO MEFs in response to IR treatment by staining the cells with
7-AAD and Annexin V-FITC. Using flow cytometric analysis, we classified four groups of cells: necrotic (quadrant 1), late apoptotic (quadrant 2), early apoptotic (quadrant 3), and live (quadrant 4). Elp1 knockout alone or in combination with IR treatment caused a marked increase in the proportion of early and late apoptotic cells relative to Ctrl cells (Fig. 3C). We obtained similar results for cells treated with ETO, with the proportion of late apoptotic cells being significantly elevated in cells lacking Elp1 upon treatment with $10 \mu \mathrm{M}$ ETO for $24 \mathrm{~h}$ compared to Ctrl cells (Additional file 1: Fig. S4). These data indicate that knockout of Elp1 results in hypersensitivity to IR- and genotoxic agent-induced DNA damage.

\section{Elp 1 is required for HR repair}

Eukaryotic cells repair DSBs mainly via the HR and NHEJ pathways. Next, we investigated a possible role for Elp1 in DSB repair using U2OS cell lines harboring reporter systems for HR or NHEJ [16]. Relative HR or NHEJ frequency was normalized to the percentage of untreated cells. I-SceI, a yeast homing endonuclease, was used to induce a DSB within an 18 base-pair (bp) sequence, causing overhangs of four nucleotides [22]. U2OS direct repeat green fluorescent protein (DR-GFP) cells that harbor a single copy of a genomically-integrated DRGFP construct were used to measure homology-directed repair (HDR). DR-GFP includes a GFP expression cassette interrupted by an I-SceI recognition site, followed by an internal GFP (iGFP) fragment (Fig. 4A). Upon DSB induction following I-SceI expression, a functional GFP gene and GFP signals can only be restored by HDR using the downstream iGFP sequence as template. We found that, in the absence of I-SceI expression, GFP signals were barely detected in these U2OS DR-GFP cells treated with control or ELP1 shRNA (Fig. 4B). In the presence of I-SceI expression, HDR was triggered in cells treated with control shRNA, as reflected by our detection of GFP signals (Fig. 4B and S5A). Conversely, knockdown of ELP1 significantly decreased the frequency of HDR upon exogenous expression of I-SceI (Fig. 4B and

\footnotetext{
(See figure on next page.)

Fig. $2 \mathrm{KO}$ MEFs accumulate elevated levels of DNA damage in response to IR and ETO treatment. A Representative images of $\mathrm{YH} 2 \mathrm{AX}$ foci staining. Elp $7^{\text {floxflox }}$ MEFs were infected with Ctrl or Cre-expressing lentivirus. Cells were either non-irradiated ( 0 h) or treated with 4 Gy of IR at D5, before being returned to normal culture conditions for a further 1, 4 or $24 \mathrm{~h}$. Cells were then fixed and an immunofluorescence assay was performed using antibodies against $\mathrm{YH} 2 \mathrm{AX}$ (green). Nuclei were stained with DAPI (blue). Scale bars, $25 \mu \mathrm{m}$. B Quantification of the number of cells with more than $10 \mathrm{YH} 2 \mathrm{AX}$-positive foci per cell. For each time-point, at least 200 cells per group were counted. Results represent the average of three independent experiments. C Representative images of cells analyzed by comet assay. Ctrl and KO MEFs were either non-irradiated (0 h) or exposed to 4 Gy of IR, and then $24 \mathrm{~h}$ later they were subjected to comet assay. Arrows indicate genomic DNA migrating away from its original location. Scale bars, $50 \mu \mathrm{m}$. D Quantification of DNA damage was performed by comet assay. We measured the parameters of \% tail DNA (left panel) and tail moment (right panel). Data shown are from three independent experiments, and at least 50 comets per group were measured per experiment. E, F Detection of DNA damage by staining for $\mathrm{YH} 2 \mathrm{AX}$ foci. Ctrl and KO MEFs were either untreated (no treatment) or treated with $40 \mu \mathrm{M}$ of ETO for 2, 16 or $24 \mathrm{~h}$, and then subjected to $\mathrm{\gamma H} 2 \mathrm{AX}$ immunofluorescence staining. Representative images are shown in (E), and $\gamma \mathrm{H} 2 \mathrm{AX}$ intensity per cell is summarized in (F). YH2AX intensity per nucleus was analyzed using Image J software. The images of at least 200 cells per group were taken and used to quantify $\mathrm{\gamma H} 2 \mathrm{AX}$ intensity. Scale bars, $10 \mu \mathrm{m}$. Data are presented as mean $\pm \mathrm{SEM} .{ }^{*} p<0.05 ;{ }^{* *} p<0.01 ;{ }^{* * *} p<0.001$ compared to Ctrl cells using Student's $t$-test
} 


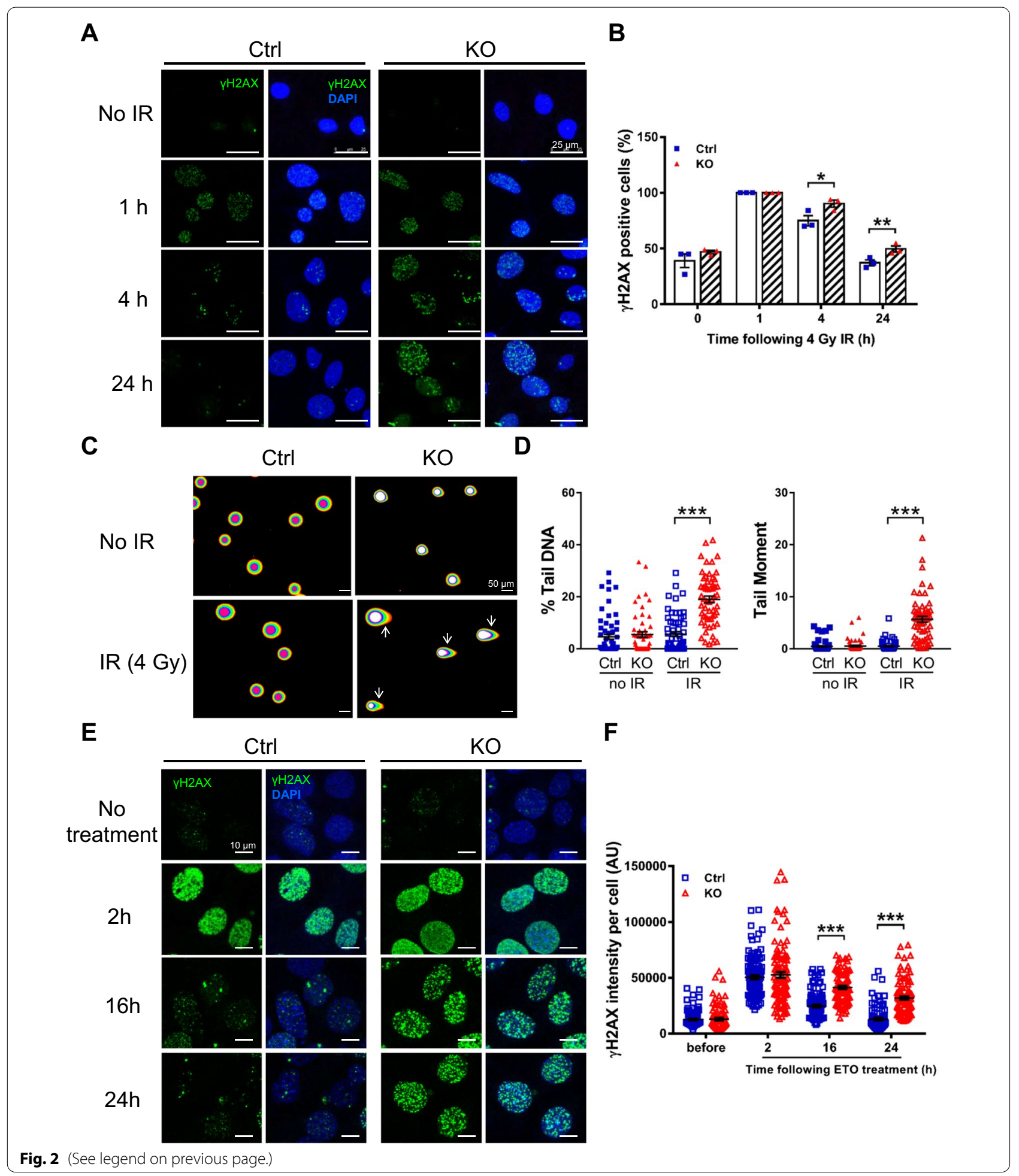

Additional file 1: Fig. S5A), suggesting that the HR repair pathway was suppressed by silencing ELP1. In addition, we employed the NHEJ reporter EJ5-GFP, which includes two fragments of a GFP expression cassette interrupted by a puro gene that is flanked by two I-SceI recognition sites [23]. GFP signal can only be restored by the NHEJ repair pathway when the puro gene is removed by exogenously expressed I-SceI (Fig. 4A) [23]. As for EJ5-GFP, 

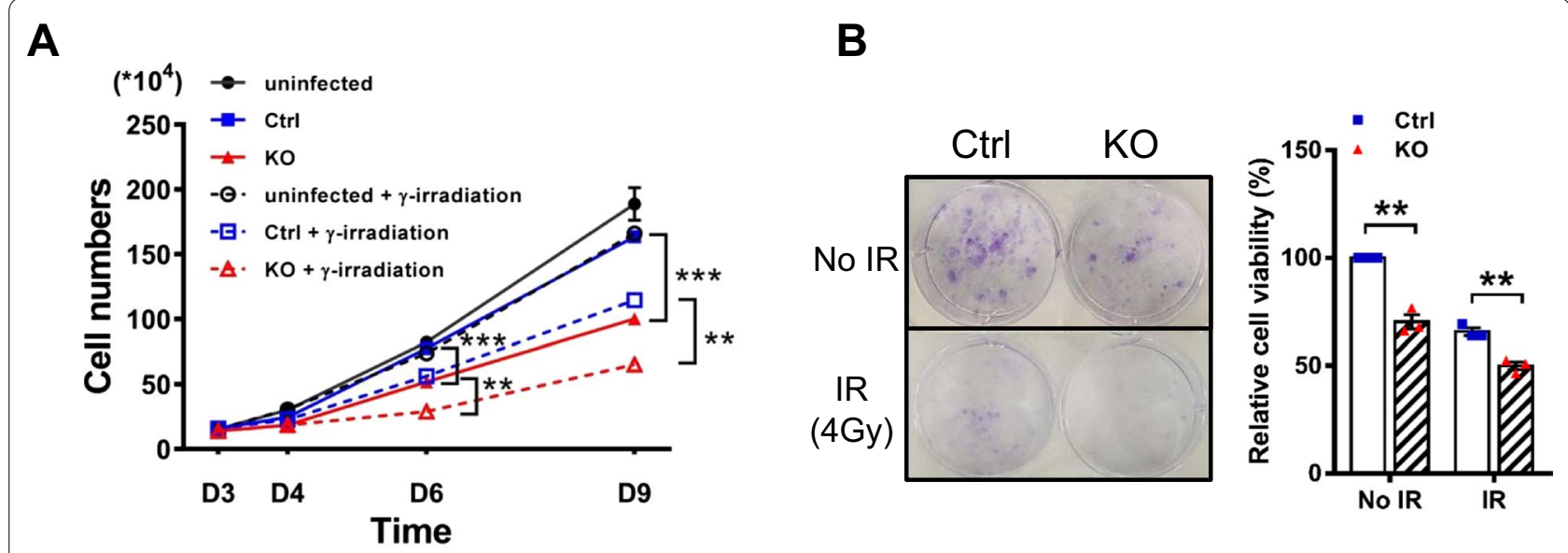

C
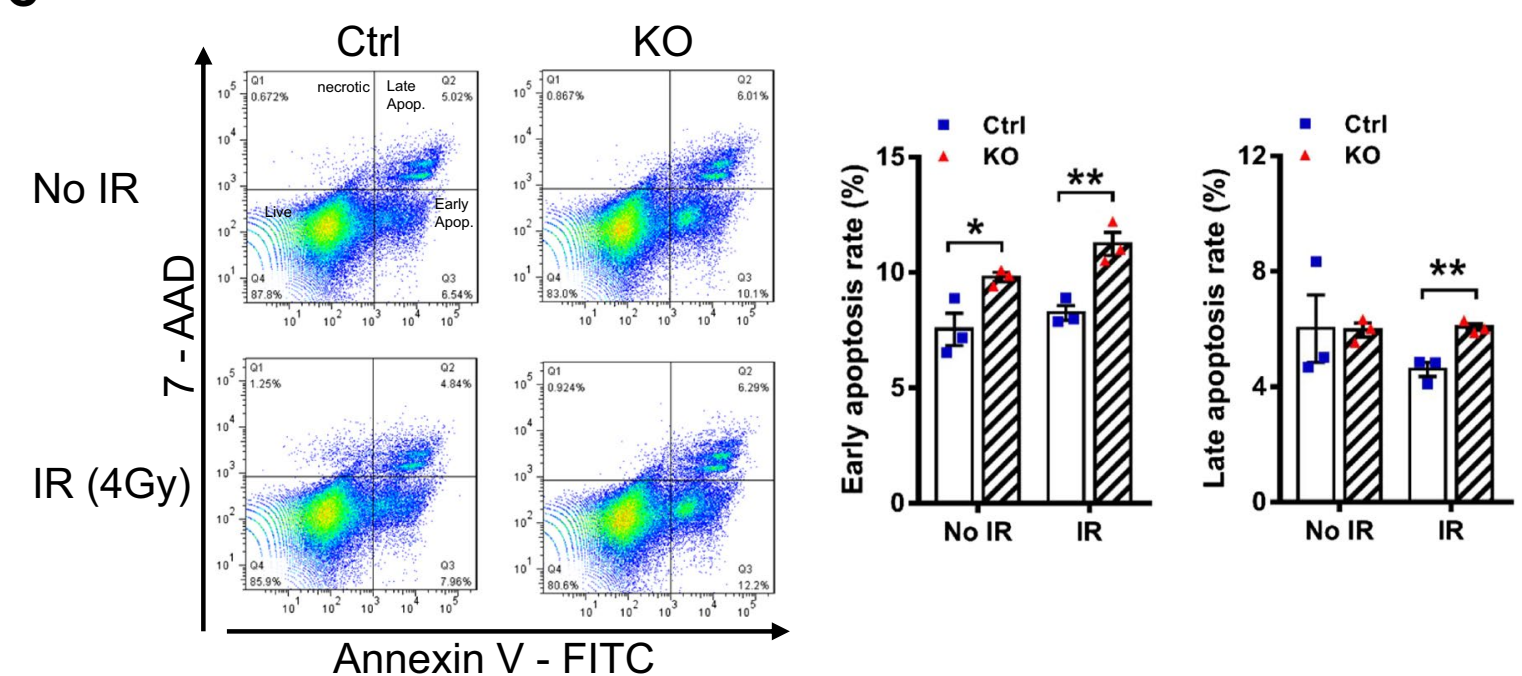

Fig. 3 Elp1 deficiency inhibits cell viability and colony formation, and induces apoptosis in MEFs in response to IR. A, B Cell numbers and clonogenic assays for Ctrl and KO MEFs that had either been non-radiated (no IR) or exposed to 4 Gy of IR at D5. A Cell numbers were counted at the indicated time-points. B At D10, cells were stained with crystal violet (left panel), and cell viability was quantified (right panel). C Analysis of apoptosis in Ctrl and KO MEFs with or without IR treatment at D10. Cells are classified as "late apoptotic" (top right quadrant), "necrotic" (top left), "live" (bottom left), or "early apoptotic" (bottom right). Representative scatter plots (left panel) and quantitative analysis (right panel) of early apoptotic cells are shown. Results represent the average of three independent experiments. Data are presented as mean \pm SEM. ${ }^{*} p<0.05 ;{ }^{* *} p<0.01$ compared to Ctrl cells using Student's t-test

we could barely detect GFP signal in Ctrl or ELP1-knockdown cells in the absence of I-SceI induction (Fig. 4C). However, unlike our results for the HR reporter assay, the frequency of NHEJ was not significantly altered by silencing ELP1 (Fig. 4C and Additional file 1: Fig. S5B). Expression of ELP1, HA-I-SecI, and $\beta$-actin in both DR-GFP and EJ5-GFP cell lines was further confirmed by Western blot analysis (Fig. 4D, E, Additional file 1: Fig. S5C, D). These results imply that ELP1 mainly affects the HR repair pathway elicited by DNA damage.

In response to DNA damage, mammalian cells activate cell cycle checkpoints, recruit DNA repair proteins, and/or trigger apoptosis. ATM and ATR are central to these responses [24]. ATM rapidly reacts to DSBs induced by agents such as IR. At the DNA damage foci, ATM phosphorylates effector molecules, such as p53 [25]. We then analyzed the activation of p53. In the absence of IR treatment, p53 phosphorylation on serine 15 was increased in KO MEFs compared to Ctrl cells, whereas the p53 protein level was not significantly altered between groups (Additional file 1: Figure S6A). Compared to non-irradiated cells, IR induced phosphorylation of p53 at serine 15 in both Ctrl and KO cells, with a significant increase in Ctrl cells. These results suggested that ATM-mediated phosphorylation of p53 was not defective upon Elp1 deficiency. DNA 


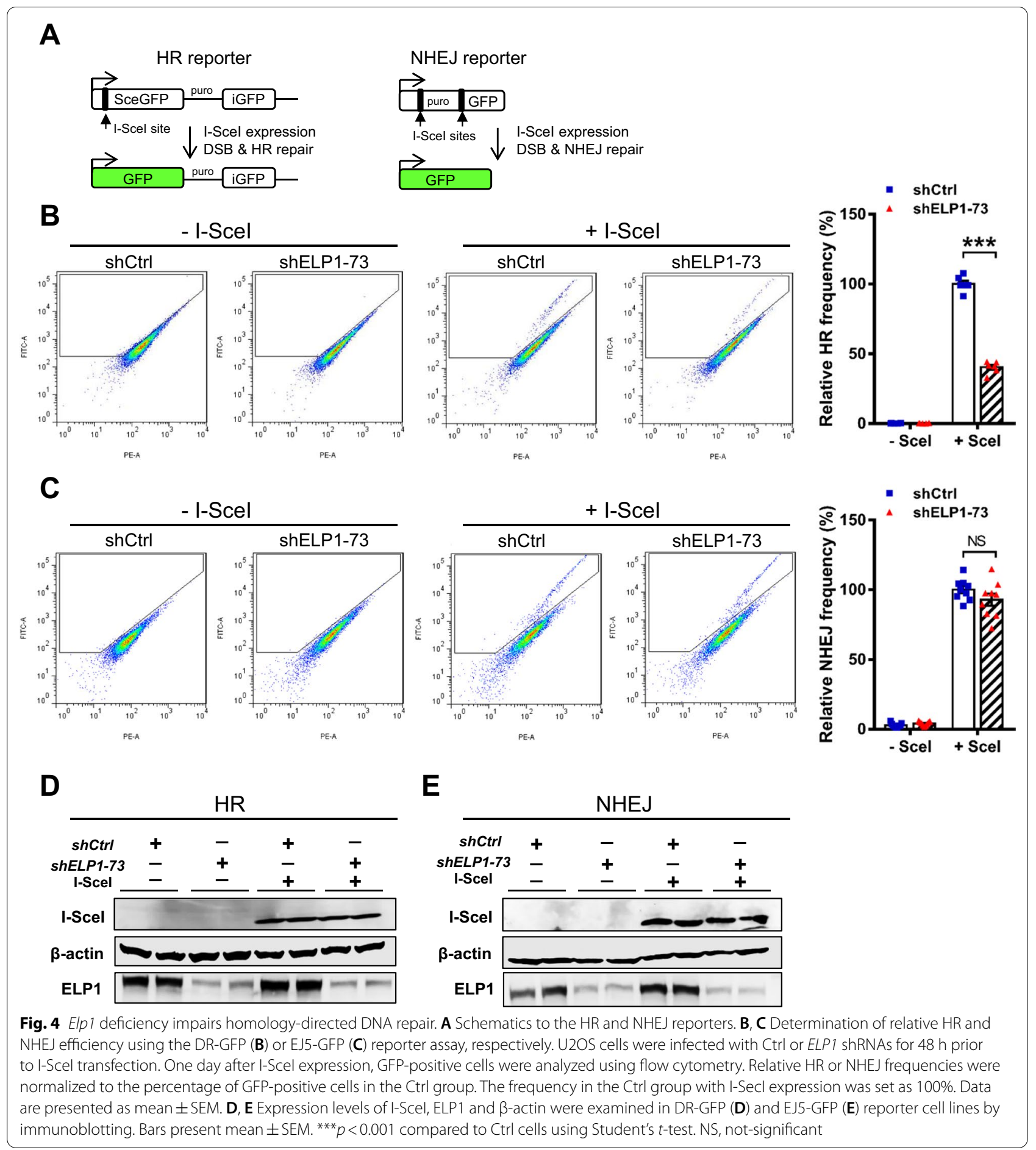

end resection, an early step of HR repair, produces $3^{\prime}$ single-stranded DNA (ssDNA) tails, and replication protein A (RPA) is the main eukaryotic ssDNA-binding protein [26]. We examined whether RPA-ssDNA complex was formed in KO MEFs by measuring RPA foci.
Cells showing the high level of foci ( $>10$ per cell) were evident as early as $1 \mathrm{~h}$ after IR treatment and continued to accumulate through $24 \mathrm{~h}$. The proportion of cells with high level of RPA foci was similar between Ctrl and KO MEFs (Additional file 1: Fig. S6B), suggesting 
that RPA coating was not significantly affected by the loss of Elp1.

\section{Elp1 regulates the translational efficiency of Rad51}

Targeting RAD51 to the resected ssDNA and formation of RAD51 filament are critical steps during the HR process. Recruitment of RAD51 to the resected ssDNA can be monitored by the appearance of RAD51 foci, which are considered markers of HR repair [27]. To confirm a role for Elp1 in promoting HR, we examined the effect of Elp1 deficiency on formation of RAD51 foci. We performed an immunofluorescence analysis of $\mathrm{KO}$ cells using anti-RAD51 antibodies before and after IR treatment. Consistent with the results of our HR reporter assay, $\mathrm{KO}$ cells displayed a significant decrease in number of RAD51 foci at 4 and $24 \mathrm{~h}$ post-irradiation relative to Ctrl cells (Fig. 5A, B). These results confirm a crucial role for Elp1 in the HR repair pathway.

Since RAD51 is a critical regulator of HR repair, we wondered if loss of Elp1 affects RAD51 protein levels. Immunoblot analysis demonstrated that although amounts of RAD51 were not affected upon knockout of Elp1 from cells in the absence of irradiation treatment $(0 \mathrm{~h})$ and $1 \mathrm{~h}$ post-irradiation, we observed a reduced amount of RAD51 protein in $\mathrm{KO}$ cells relative to $\mathrm{Ctrl}$ cells at 4 and $24 \mathrm{~h}$ post-irradiation (Fig. 5C, D). Since ELP1 was implicated in transcription elongation [8], we wondered if knockout of Elp1 affects Rad51 mRNA levels. In the absence of IR, Rad51 mRNA levels were comparable between Ctrl and KO cells (Fig. 5E), suggesting that Rad51 transcription was unlikely to be regulated by ELP1. Intriguingly, Ctrl cells displayed a significant increase in $\operatorname{Rad} 51$ mRNA expression at $4 \mathrm{~h}$ post-irradiation relative to $\mathrm{KO}$ cells.

Mammalian Elp1 also functions in tRNA modifications [14], which may impact protein synthesis. To assess if Elp1 affects translation of Rad51, we performed a polysome profiling analysis, enabling us to separate translating mRNAs on a sucrose gradient based on amounts of associated ribosomes. The number of ribosomes associated with an mRNA can be used as a measure of the translation state of that mRNA. Generally, mRNAs found in the non-polysomal fraction of cells are considered nontranslated. mRNAs found in the low-molecular-weight
(LMW) polysomal fraction present a moderate translational efficiency, whereas those found in the high-molecular-weight (HMW) polysomal fraction exhibit a high translational efficiency [28]. We calculated the translation status of Rad51 mRNA based on relative levels in each polysomal fraction. We found overall polysome abundance was similar between Ctrl and $\mathrm{KO}$ cells in the presence or absence of irradiation treatment (Fig. $5 \mathrm{~F}$ and Additional file 1: Fig. S7), suggesting that loss of Elp1 does not globally alter protein translation. Next, we analyzed the translational status of Rad51 mRNA specifically. In the absence of irradiation, Elp1 knockout did not significantly change the polysome distribution pattern of Rad51 and Gapdh mRNAs (Fig. 5G). Notably, upon irradiation treatment, knockout of Elp1 from MEFs caused Rad51 mRNA to be distributed in the non-polysomal fraction, whereas the majority of Gapdh mRNA in both $\mathrm{Ctrl}$ and $\mathrm{KO}$ cells remained associated with high-molecular-weight (HMW) polysomes (Fig. 5G). We also examined the distribution of Brca2 and Xrcc4 mRNAs in the polysomal fractions. Breast cancer 2 (BRCA2) has been shown previously to interact with RAD51 and mediates RAD51-dependent DNA repair [29, 30]. DNA repair protein 4 (Xrcc4) is one of several core proteins involved in the NHEJ pathway for repairing DSBs [31]. The polysome distribution profiles of both Brca2 and Xrcc4 mRNAs were not significantly altered between Ctrl and KO cells upon IR treatment (Additional file 1: Fig. S8). These results indicate that Elp1 knockout decreases the translational efficiency of Rad51 mRNA upon IR exposure without affecting the translation of some other mRNAs.

\section{Ectopic RAD51 expression partially rescues impairment of DNA damage repair in Elp1-knockout MEFs}

If RAD51 mediates the role of Elp1 in regulating DNA damage repair, we predicted that ectopic expression of RAD51 in KO MEFs might rescue their defects in DSB repair. To explore that possibility, we infected MEFs with control or Cre lentiviruses to induce Elp1 deletion and then transfected the cells with control or human RAD51expressing vectors. Comet assays were then performed in the irradiated or non-irradiated cells. Consistent with our above-described results, we found that deletion of Elp1 significantly increased DNA tail length upon IR

\footnotetext{
(See figure on next page.)

Fig. 5 Elp1 deficiency impairs formation of RAD51 foci by regulating RAD51 protein levels and Rad51 translation. A Representative images of RAD51 foci in Ctrl and KO MEFs without irradiation ( $0 \mathrm{~h}$ ) or 1, 4 or $24 \mathrm{~h}$ post-irradiation. Scale bars, $10 \mu \mathrm{m}$. B Quantification of the number of RAD51 foci per cell, with at least 100 cells counted for each independent experiment. C, D Western blotting analysis (C) and quantitative analysis (D) of RAD51 protein levels. $\beta$-actin was used as a loading control. E qRT-PCR analysis of Rad51 mRNA in Ctrl and KO MEFs that were either non-radiated (0 h) or exposed to $4 \mathrm{~Gy}$ of IR. Data are from three independent experiments. Results are presented as means \pm SEM. Student's $t$-test: ${ }^{*} p<0.05 ;{ }^{* *} p<0.01$; ${ }^{* * *} p<0.001$. F Polysomes from Ctrl and KO MEFs without irradiation ( $0 \mathrm{~h}$ ) (top panel) or $4 \mathrm{~h}$ post-irradiation (4 Gy) (lower panel) were fractionated by sucrose gradient centrifugation for polysome profile analyses. G qRT-PCR analysis of Rad51 and Gapdh mRNAs in polysomal fractions from (F). The translational activity associated with each fraction is indicated as: non-polysome (not translated); LMW-low-molecular-weight polysomes (moderately translated), or HMW—high-molecular-weight polysomes (actively translated)
} 
A

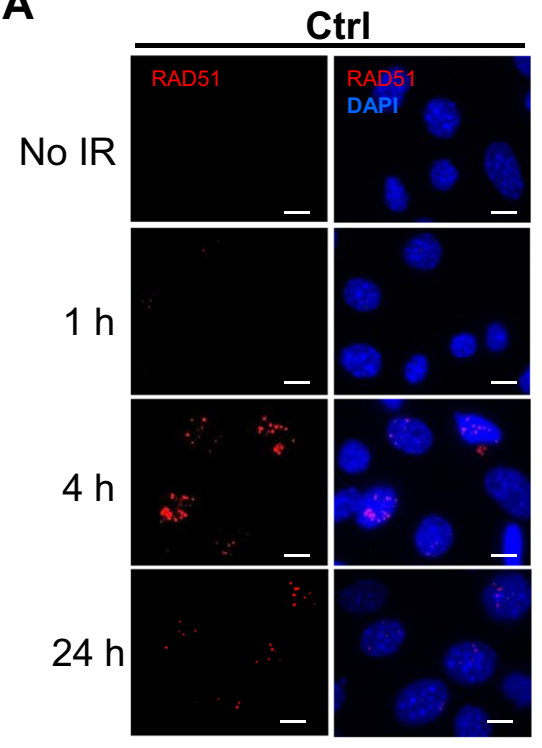

E

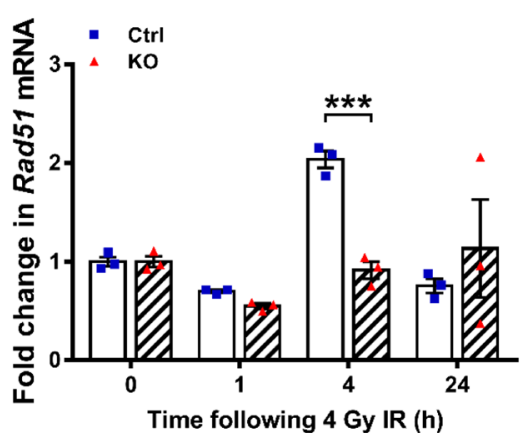

$\mathbf{F}$

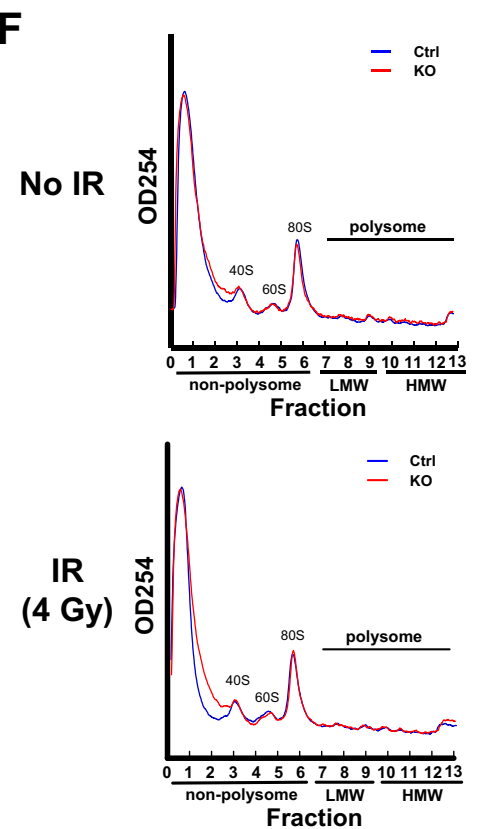

Fig. 5 (See legend on previous page.)
B
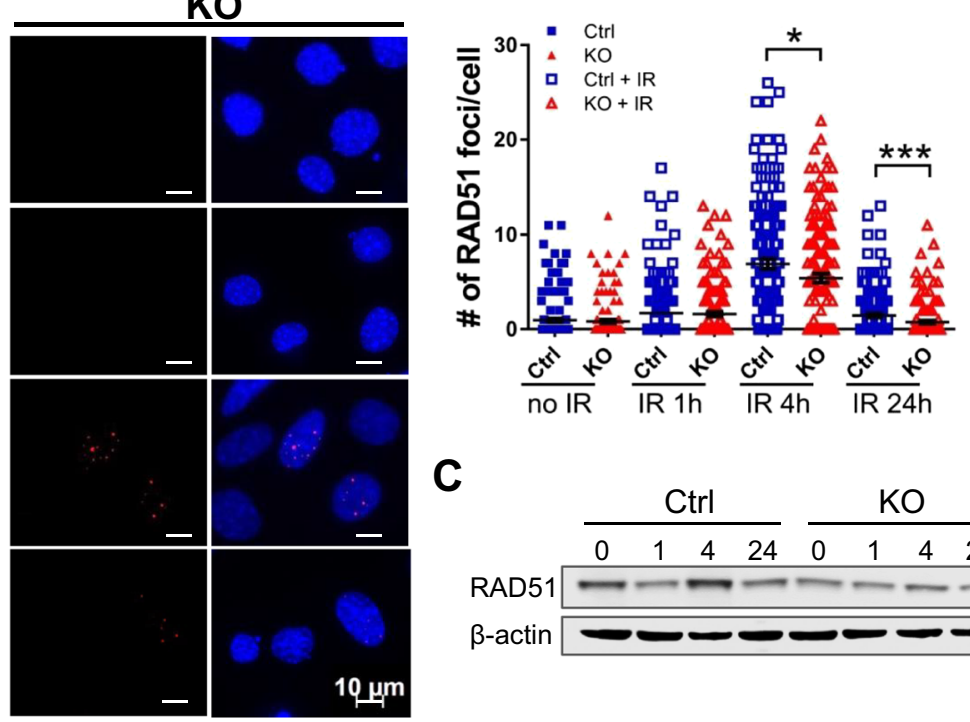

C

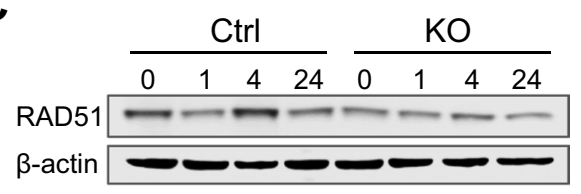

D : ctrr $^{2}$
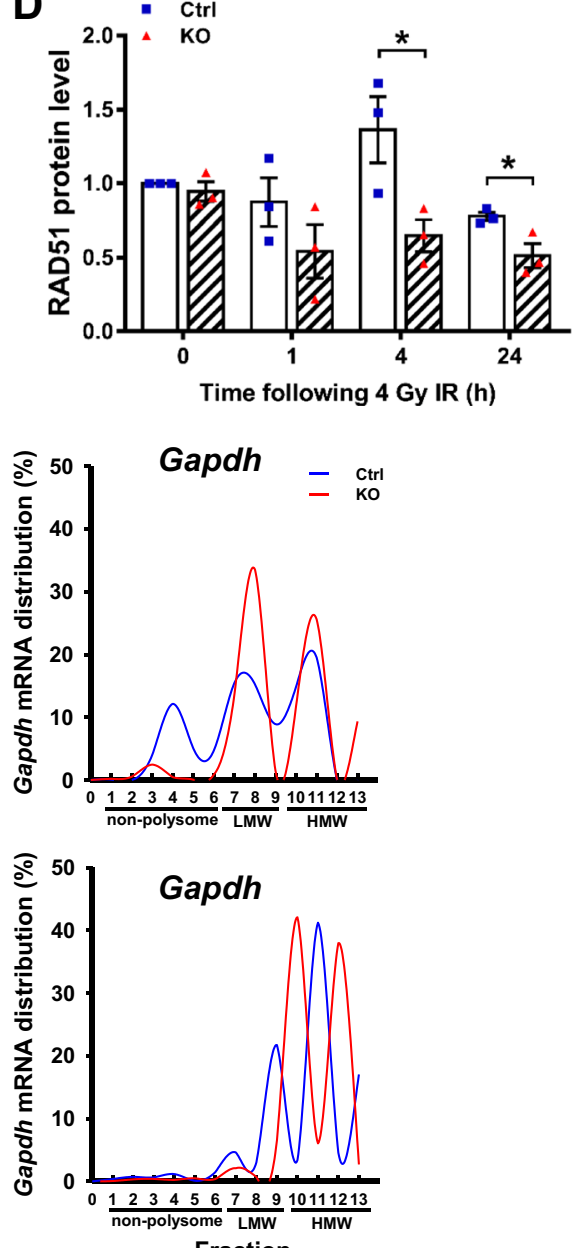
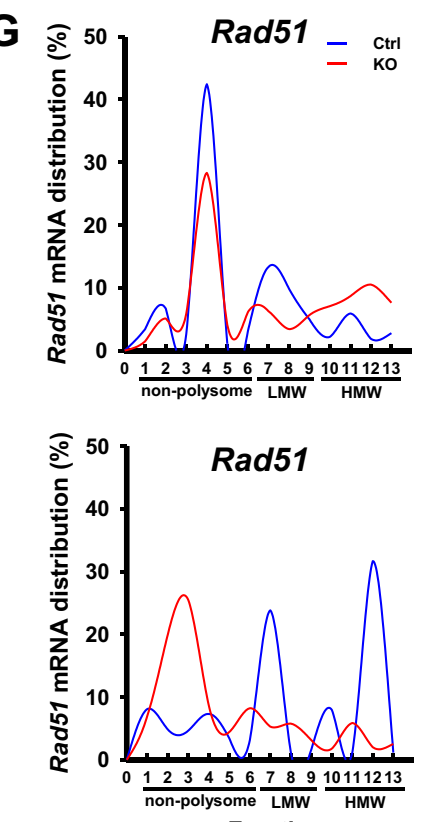

Fraction

Fraction 
A

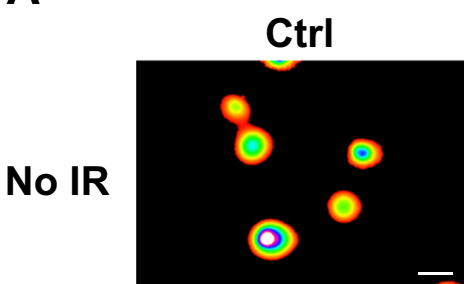
Ctrl + RAD51

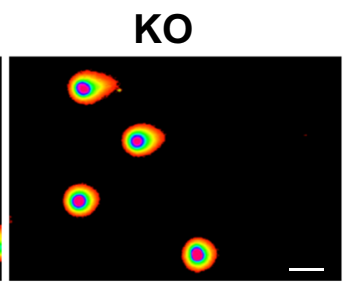

KO + RAD51
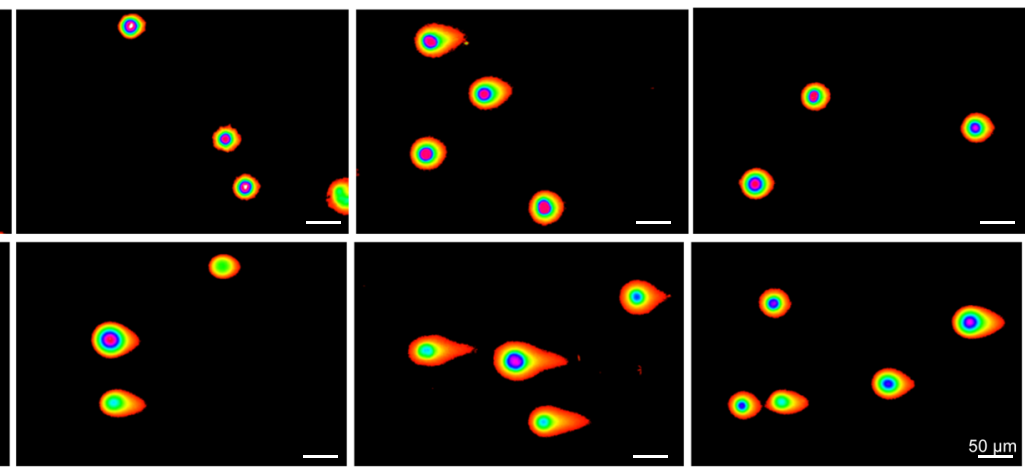

B

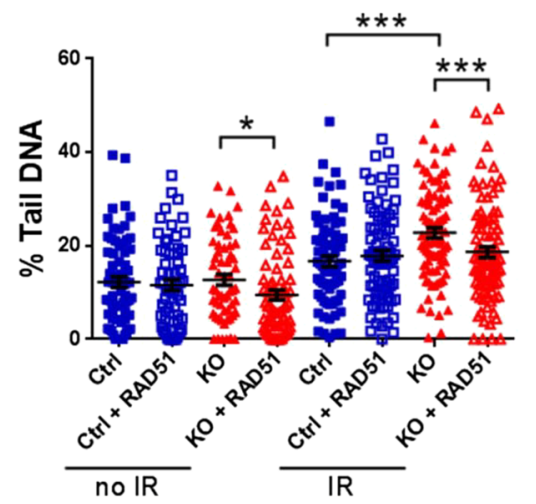

Fig. 6 Ectopic expression of RAD51 in KO MEFs abrogates radiation-induced genomic fragmentation. A The effects of human RAD51 overexpression on DNA damage were examined in Ctrl and KO MEFs with or without IR treatment by means of comet assay. Scale bars, 50 um. B Quantification of \% tail DNA. Data shown are from three independent experiments, and at least 100 comets per group were measured in each experiment. Data are presented as mean \pm SEM. Student's $t$-test: ${ }^{*} p<0.05 ;{ }^{* *} p<0.01 ;^{* * *} p<0.001$. Scale bars, $50 \mu \mathrm{m}$

treatment relative to $\mathrm{Ctrl}$ cells, indicative of their having more DNA breaks (Fig. 6A, B). More importantly, ectopic expression of RAD51 in KO MEFs partially rescued IRinduced genomic DNA fragmentation (Fig. 6A, B), suggesting that Elp1 mediates DSB repair at least in part by modulating the expression of RAD51. Western blot analysis confirmed the ectopic expression of RAD51 in MEFs, and we found no influence of ectopic RAD51 expression on ELP1 levels (Additional file 1: Fig. S9).

\section{Important signaling pathways are associated with Elp1 deficiency}

To elucidate the mechanisms of Elp1-mediated IRinduced DSB repair in MEFs, we conducted comprehensive profiling on the proteins isolated from the Ctrl and KO MEFs treated with or without IR ( 0 or $4 \mathrm{~h}$, respectively). We identified a total of 5753 proteins by LC-MS/ MS. Normalized abundance values of the housekeeping protein GAPDH were comparable among groups
(Additional file 1: Fig. S10). The normalized abundance value of $\beta$-actin was slightly higher in $\mathrm{KO}$ than Ctrl MEFs (Additional file 1: Fig. S10). Our proteomic profiling analysis revealed a total of 901 or 569 significant changes in proteins abundance (fold-change $\leq 0.67$ or $\geq 1.5$ ) between $\mathrm{Ctrl}$ and $\mathrm{KO}$ cells at the 0 time-point or between $\mathrm{Ctrl}$ and $\mathrm{KO}$ cells at the $4 \mathrm{~h}$ time-point, respectively, representing a $10-16 \%$ alteration from the total identified proteome.

We further examined the differentially expressed proteins according to biological processes using Ingenuity software for IPA. Of the 901 proteins identified from non-irradiated MEFs ( $0 \mathrm{~h}$ time-point), 247 were up-regulated (27\%) and 654 were down-regulated (73\%) (Fig. 7A). The cell localizations of these 901 proteins were primarily linked to cytoplasm $(n=496,55 \%)$, nucleus $(n=211$, $23 \%)$, plasma membrane $(n=95,11 \%)$, and extracellular space $(n=60,7 \%)$ (Fig. 7A). Of the 569 differentially expressed proteins in irradiated cells ( $4 \mathrm{~h}$ time-point), 


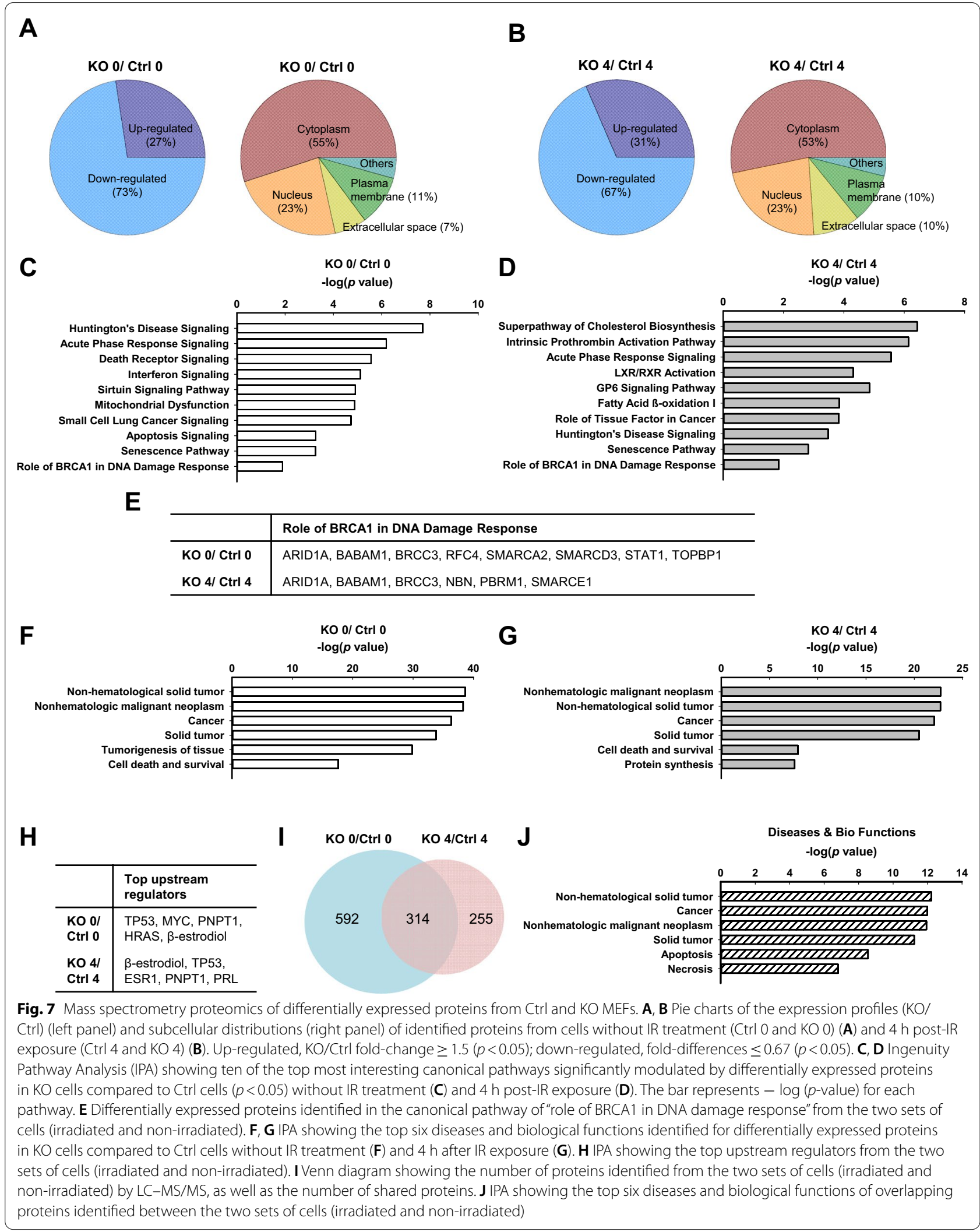


179 were up-regulated $(31 \%)$ and 390 were down-regulated (69\%) (Fig. 7B), and their principle localization were in cytoplasm $(n=303,53 \%)$, nucleus $(n=130,23 \%)$, plasma membrane $(n=59,10 \%)$, and extracellular space $(\mathrm{n}=55,10 \%)$ (Fig. 7B).

IPA also revealed several important cancer-related signaling pathways that are differentially impacted in irradiated and non-irradiated cells. Our interested top ten common canonical pathways associated with Elp1 deficiency include Huntington's disease signaling, acute phase response signaling, senescence pathway, and role of BRCA1 in DNA damage response (Fig. 7C). These same four pathways were also identified in KO MEFs subjected to IR treatment (Fig. 7D). Although RAD51 did not appear among proteins in the pathway underlying the "role of BRCA1 in DNA damage response", we did identify ARID1A, BABAM1, and BRCC3 as being differentially expressed in both sets of MEFs (Fig. 7E). Protein expression levels were retrieved from MS results and protein expression ratio (relative to Ctrl) of ARID1A, BABAM1, and BRCC3 in non-irradiated MEFs ( $0 \mathrm{~h}$ time-point) was $2.7,0.29$, and 0.38 , while their ratio in irradiated cells $(4 \mathrm{~h}$ time-point) was $0.56,1.56$, and 0.28 . We further analyzed their mRNA levels using qRT-PCR. In the presence or absence of IR, Arid1a, Babam1, and Brcc3 mRNA levels were not significantly altered between $\mathrm{Ctrl}$ and $\mathrm{KO}$ cells, indicating that their transcription was unlikely to be regulated by ELP1 (Additional file 1: Fig. S11A). Moreover, in the absence of irradiation, knockout of Elp1 from MEFs caused more Babam1 and Brcc3 mRNA to be distributed in the LMW and non-polysomal fractions (Additional file 1: Fig. S11B). In response to IR treatment, similar result on Arid1a mRNA distribution was obtained, whereas comparable or more Babam1 and Brcc3 mRNA was associated with HMW polysomes (Additional file 1: Fig. S11B). These results suggested that in non-irradiated MEFs, knockout of Elp1 decreases the translational efficiency of Babam1 and Brcc3 mRNA.

We also performed disease and biological function analysis on these proteins, and found that important differentially-expressed proteins associated in common with Elp1 deficiency and/or IR treatment included non-hematological solid tumor, non-hematologic malignant neoplasm, cancer, and solid tumor (Fig. 7F, G). In particular, "protein synthesis" which encompasses 84 proteins, was resolved as one of the top diseases and functions for differentially expressed proteins in the irradiated cell group (Fig. 7G). Details of these selected common alterations of "disease and function" in the paired sets of MEFs are summarized in Additional file 1: Tables S2, S3. Statistically significant upstream regulators in both sets of MEFs included tumor protein 53 (TP53) and polyribonucleotide nucleotidyltransferase 1 (PNPT1) (Fig. 7H). Between the two paired treatments, a total of 314 proteins were found to be overlapped (Fig. 7I). A total of 314 proteins were shared across the two sets of proteins (i.e. nonirradiated and irradiated) (Fig. 7I), which could be linked to cancer, apoptosis, and necrosis (Fig. 7J). Overall, our findings indicate that cancer pathways are the most common feature associated with Elp1 deficiency.

\section{Discussion}

Here, we provide multiple lines of evidence demonstrating a critical role for Elp1 in maintaining genomic stability and facilitating DNA repair. Deletion of Elp1 enhanced genomic instability, as evidenced by the elevated rate of chromosome breakage upon IR treatment. KO MEFs are hypersensitive to IR and chemicals that induce DSBs. In addition, together with the results of our HR reporter assay, the reduced RAD51 protein levels and a diminished capability to form RAD51 foci of KO MEFs strongly imply that Elp1 promotes HR-dependent DSB repair.

With respect to the mechanism underlying the stimulatory role of Elp1 in HR repair, our findings unveil that Elp1 potentially promotes RAD51 protein levels by controlling its translation, consequently enhancing RAD51 foci formation at DSBs. Ectopic expression of RAD51 in KO MEFs partially inhibited comet tail formation, implying that Elp1 promotes HR repair in a RAD51-dependent manner. RAD51 plays a central role in HR-mediated DSB repair. Disruption of Rad51 in mice was shown previously to induce early embryonic lethality [32]. Rad51defective cells were found to exhibit chromosome breaks and cell death [33], suggesting a role for RAD51 in cell survival. Intriguingly, RAD51 is overexpressed in many tumors that present aberrantly increased HR activity [34]. Thus, tight regulation of RAD51 is required to maintain genomic integrity. Our results reveal a novel mechanism by which RAD51 protein levels are regulated in response to DNA damage.

ELP1 was initially purified and described as an RNA polymerase II-associated transcription elongation factor [8]. However, the predominantly cytoplasmic localization of ELP1 has prompted an intense investigation of its functions apart from nuclear transcription. It has been subsequently reported that the Elongator complex is involved in translation due to its crucial involvement in modifications, including a thiol $\left(\mathrm{S}^{2}\right)$ and a methoxycarbonyl-methyl $\left(\mathrm{mcm}^{5}\right)$, at the wobble position $\left(\mathrm{U}_{34}\right)$ of certain tRNAs in fission yeast, nematode, mouse, and human $[7,14,35]$. The $\mathrm{mcm}^{5} \mathrm{~S}^{2}$ modification to $U_{34}$ enhances the translational efficiency of the AA-ending codons (i.e., AAA, GAA, CAA) [36, 37], whereas its impact on the translation of the AG-ending codons remains largely unclear. There is now widespread consensus that the 
Elongator complex has an evolutionarily conserved role in regulating the translation efficiency of a vast number of transcripts [38, 39]. In support of that notion, we showed herein by means of a polysome profiling assay that ELP1 modulates Rad51 mRNA translation efficiency in response to IR treatment. Using a proteome-wide approach, we identified proteins that are biologically significant in the response to Elp1 knockout, and those proteins were enriched in several significant pathways such as cancer, apoptosis signaling, protein synthesis, and DNA damage response. We and others' findings support a critical role for Elp1 in translational regulation. Studies in fission yeast and mouse have shown that Elongator mutants exhibit reduced levels of proteins encoded by large ( $\geq 1755$ total codons), AA-biased transcripts [3841]. We then analyzed the codon usage of mouse Rad51 transcript (NM_011234). Interestingly, we found that mouse Rad51 transcript encodes 339 amino acids, and is AG-biased (the number of AA-ending codons over the number of AG-ending codons, or the AA:AG ratio=0.9). Although some studies report that the targets of Elongator are large, and enriched in AA-ending (wobble) codons $[38,39]$, in agreement with our finding, elp3 mutant yeast exhibited a small decrease in ribosome density at AGending codons [42]. Further studies will be necessary to examine whether and how the loss of Elongator complex affects the pairing between $\mathrm{U}_{34}$ and G-ending codons, and whether RAD51 is directly regulated by ELP1.

Another surprising finding that emerged from our study is that not only Rad51 translation but also $\operatorname{Rad} 51$ transcription was considerably affected by the combination of Elp1 knockout plus IR treatment. Knockout of Elp1 did not affect Rad51 transcription in the absence of IR. In contrast, a robust increase in $\operatorname{Rad} 51$ mRNA was observed in Ctrl cells, but not in KO cells upon IR treatment, suggesting that other ELP1-dependent factor(s) promote Rad51 transcription. Previous studies have shown that $\operatorname{Rad} 51$ transcription is positively regulated by the transcription factor EGR1 and members of the E2F transcription factor family [43, 44], but it is negatively regulated by $\mathrm{p} 53$ and hypoxia $[45,46]$. However, neither the EGR1 nor E2F transcription factors were identified as differentially expressed in our KO MEFs. There are two possibilities for that outcome. First, the EGR1 and E2F transcription factors may not be regulated by ELP1. Second, the change in expression levels of these factors is below the detection levels achievable by LC-MS/MS method. It would be interesting to validate the protein levels of the EGR1 and E2F transcription factors using Western blot analysis. Although we cannot exclude the possibility that some nuclear ELP1 directly regulates Rad51 mRNA expression, our results favor the cytoplasmic localization of ELP1 modulates the translation of certain transcriptional regulators that might control Rad51 mRNA expression. Moreover, apart from transcriptional and translational controls, we did not rule out the possibility that ELP1 may regulate $\operatorname{Rad} 51$ mRNA stability.

Using polysome profiling analysis, we demonstrated an altered distribution of the majority of $\operatorname{Rad} 51 \mathrm{mRNA}$ from the non-translated to highly translated fractions in Ctrl MEFs in response to IR treatment. Meanwhile, we also noticed a burst in $\operatorname{Rad} 51$ transcription in irradiated Ctrl MEFs compared to non-irradiated MEFs. It is possible that during this time, newly synthesized Rad51 transcripts in Ctrl MEFs are modified differently such that they are more stable, more likely to be recognized and picked up by polysomes. Nevertheless, under most conditions, translational efficiency is proportional to the number of ribosomes associated with an mRNA [47]. In the context of total Rad51 mRNA within the cells, its translation efficiency was reduced in KO MEFs upon IR treatment.

The 314 proteins shared between the two sets of MEFs (irradiated or non-irradiated) were involved in various functions including cancer, cell death and survival, and organismal survival. Strikingly, around 95\% $(n=301)$ of them were mapped to cancer pathways, evidencing a strong link between Elp1 deficiency and cancer. Unexpectedly, RAD51 was not identified among the differentially expressed proteins between Ctrl and $\mathrm{KO}$ cells. There are two possibilities for this outcome, including incomplete Elp1 knockout and the presence of abundant RAD51 proteins/peptides of similar molecular weight. First, the normalized abundance value of ELP1 was only reduced by $30 \%$ in our KO cells (data not shown), potentially causing an undetectable change in RAD51 protein levels. Second, the presence of abundant proteins/ peptides of similar molecular weight to RAD51 might have prevented us from detecting RAD51. Even though RAD51 was not identified among the mapped canonical pathways, three proteins (ARID1A, BABAM1, and BRCC3) involved in the pathway for "role of BRCA1 in DNA Damage Response" were shared between the two sets of MEFs. ARID1A (AT rich interactive domain 1A), one of the SWI/SNF chromatin remodeling complexes, facilitates efficient DNA DSB end resection and it sustains ATR-dependent DNA damage signaling [48]. Both BABAM1 (BRISC and BRCA1 A complex member 1, also known as MERIT40) and the deubiquitinylating enzyme BRCC3 (BRCA1/BRCA2-containing complex subunit 3) belong to BRCA1-A complex $[49,50]$, which mediates DNA repair through HR upon DNA damage. BRCA1 can also form a complex with BRCA2/PALB2/RAD51, which promotes efficient HR-mediated repair [30, 51]. We also showed that in the absence of IR treatment, knockout of 
Elp1 did not affect transcription, but decreased the translational efficiency of Babam1 and Brcc3 mRNA. Further studies will be necessary to examine whether these proteins are directly or indirectly regulated by Elp1 at translational and/or post-translational level. Together, it is likely that the dysregulation of ARID1A, BRCC3, BABAM1, and RAD51 in KO MEFs can negatively impact genomic stability.

The disease FD is caused by reduced ELP1 protein levels in neuronal tissues due to a homozygous splice site mutation in the ELP1 gene, and it is characterized by sympathetic nerve system dysfunction $[9,10]$. The neurodegenerative hallmarks of FD as patients age include progressive gait ataxia and death of retinal ganglion cells [52]. Progressive neuronal apoptosis has also been observed in sympathetic and sensory ganglia upon neurons innervating target tissues in a mouse model of FD [53]. In addition, elevated DNA damage and misregulation of multiple DNA damage response genes was observed in dorsal root ganglia (DRG) of peripheral nervous system (PNS)-specific Elp1 knockout embryos [38]. In agreement with these studies, our proteomic results have indicated that the apoptosis pathway and DNA damage response are two of the most affected canonical pathways upon Elp1 deficiency in MEFs. Our finding regarding to elevated levels of fragmented DNA in KO MEFs relative to Ctrl MEFs upon IR treatment was slightly different from the published data from Goffena et al., who showed that Elp1 conditional knockout neurons displayed increased levels of DNA damage in the absence of IR treatment [38]. The discrepancy may be due to the cell type difference and/or that the power of our comet assay may not be strong enough to detect the minor differences. DNA damage induces cell cycle arrest and apoptosis. Thus, an accumulation of damaged DNA over time could contribute to impaired cell growth and survival. Our results support Elp1 playing a general role in the maintenance of genome integrity.

\section{Conclusions}

Elp1 maintains genomic stability by promoting HR-mediated DNA repair in a RAD51-dependent manner. We believe accumulation of DNA damage as a consequence of defective HR repair is the mechanism underlying increased genomic instability in $\mathrm{KO}$ cells.

\footnotetext{
Abbreviations

DDR: DNA damage response; DMEM: Dulbecco's Modified Eagle's Medium; DR-GFP: Direct repeat green fluorescent protein; DSBs: Double-strand breaks; ETO: Etoposide; HDR: Homology-directed repair; HMW: High-molecularweight; HR: Homologous recombination; IPA: Ingenuity pathway analysis; IR: lonizing radiation; LC-MS/MS: Liquid chromatography-tandem mass spectrometry; LMW: Low-molecular-weight; MEFs: Mouse embryonic fibroblasts; MNi: Micronuclei; MS: Mass spectrometry; NBUDs: Nuclear buds; NHEJ:
}

Non-homologous end-joining; ssDNA: Single-stranded DNA; TP53: Tumor protein 53.

\section{Supplementary Information}

The online version contains supplementary material available at https://doi. org/10.1186/s12929-021-00773-z.

\begin{abstract}
Additional file 1. Elp 1 facilitates RAD51-mediated homologous recombination repair via translational regulation. Figure S1. Schematic of the Elp 1 mutant allele. Figure S2. qRT-PCR analysis of the Elp1 mRNA expression by D3 MEFs. Figure S3. Knockout of Elp 1 impaired cell cycle. Figure S4. Apoptosis analysis of Ctrl and KO MEFs treated with ETO for $24 \mathrm{~h}$ via flow cytometry. Figure S5. Elp1 deficiency impairs homology-directed DNA repair. Figure S6. The ratio of phospho-p53/p53 protein level and the number of RPA foci were not significantly affected in the absence of Elp1. Figure S7. Part of polysome profiling of Ctrl and KO MEFs shown in Fig. 5F. Figure S8. qRT-PCR analysis of Brca2 and Xrcc 4 mRNA in polysomal fractions shown in Fig. 5F. Figure S9. Detection of RAD51, GAPDH and ELP1 by Western blot of Ctrl and KO MEFs transfected with vector expressing full-length human RAD51. Figure S10. Normalized abundance values of GAPDH and $\beta$-actin among four groups. Figure S11. qRT-PCR analysis of Arid 1a, Babam 1 and Brcc3 mRNA in Ctrl and KO MEFs and in polysomal fractions shown in Fig. 5F. Table S1. The primer sequences for qRT-PCR. Table S2. Ingenuity disease and function analysis of mapped differentially expressed proteins between $\mathrm{KO} 0$ and CtrlO cells. Table S3. Ingenuity disease and function analysis of mapped differentially expressed proteins between $\mathrm{KO} 4$ and $\mathrm{Ctr} / 4$ cells.
\end{abstract}

\section{Acknowledgements}

We would like to thank Dr. Yi Zhang for Elp $1^{\text {floxflox }}$ mice and Dr. Jeremy M Stark for reporter cell lines. We thank the National RNAi Core Facility at Academia Sinica in Taiwan for providing shRNA reagents and related services. We are grateful to Technology Commons, College of Life Science, National Taiwan University (NTU) for the cell analyzer service. We thank NTU Consortia of Key Technologies and the NTU Instrumentation Center for mass spectrometry technical research services. We are grateful to the National Laboratory Animal Center (NLAC), NARLabs, Taiwan, for technical support in contract breeding and testing services. We thank Drs. Zee-Fen Chang and Hui-Ching Wang for technical assistance. We are grateful to Drs. Chun-Ming Chen and Ching-Shyi Wu for anti-p53 and anti-RPA70 antibodies, respectively. We appreciate our laboratory members for expert mouse husbandry and assistance.

\section{Authors' contributions}

W-TC, H-YT, C-LJ, C-YL and F-JL conceived, designed, performed the experiments, analyzed the data and created the figures. PC, L-YC, K-YL and F-JL contributed to interpretation of data. PC, L-YC and I-CW provided reagents. F-JL wrote the manuscript and supervised the research. All authors read and approved the final manuscript.

\section{Funding}

This work was supported by the Ministry of Science and Technology, Taiwan [MOST 104-2320-B-002-040- and MOST 107-2320-B-002-004-MY3 to F-J.L., MOST 108-2321-B-002-054 to P.C., MOST 108-2628-B-001-006 to L-Y.C.].

\section{Availability of data and materials}

The MS data obtained in this work have been deposited to the ProteomeXchange Consortium via the PRIDE partner repository with the dataset identifier PXD024628. The rest of data generated in this study are available from corresponding author on reasonable request.

\section{Declarations}

Ethics approval and consent to participate All animal procedures were approved by the Institutional Animal Care and User Committee (IACUC) of National Taiwan University, Taiwan (NTU106-EL-00128). 


\section{Consent for publication \\ Not applicable.}

\section{Competing interests}

The authors declare that they have no competing interests.

\begin{abstract}
Author details
${ }^{1}$ Department of Biochemical Science and Technology, National Taiwan University, No.1, Sec.4, Roosevelt Rd., Taipei 10617, Taiwan. ${ }^{2}$ Institute of Biochemical Sciences, National Taiwan University, Taipei 10617, Taiwan. ${ }^{3}$ Institute of Biological Chemistry, Academia Sinica, Taipei 11529, Taiwan. ${ }^{4}$ Institute of Molecular Biology, Academia Sinica, Taipei 11529, Taiwan. ${ }^{5}$ Department of Agricultural Chemistry, National Taiwan University, Taipei 10617, Taiwan. ${ }^{6}$ Institute of Biotechnology, National Tsing Hua University, Hsinchu 30013, Taiwan. ${ }^{7}$ Research Center for Development Biology and Regenerative Medicine, National Taiwan University, Taipei 10617, Taiwan.
\end{abstract}

Received: 27 April 2021 Accepted: 2 November 2021 Published online: 24 November 2021

\section{References}

1. Lord CJ, Ashworth A. The DNA damage response and cancer therapy. Nature. 2012;481(7381):287-94.

2. Moynahan ME, Jasin M. Mitotic homologous recombination maintains genomic stability and suppresses tumorigenesis. Nat Rev Mol Cell Biol. 2010;11(3):196-207.

3. Lieber MR. NHEJ and its backup pathways in chromosomal translocations. Nat Struct Mol Biol. 2010;17(4):393-5

4. Luo SC, Yeh HY, Lan WH, Wu YM, Yang CH, Chang HY, et al. Identification of fidelity-governing factors in human recombinases DMC1 and RAD51 from cryo-EM structures. Nat Commun. 2021;12(1):115.

5. Greene EC. DNA sequence alignment during homologous recombination. J Biol Chem. 2016:291(22):11572-80.

6. San Filippo J, Sung P, Klein H. Mechanism of eukaryotic homologous recombination. Annu Rev Biochem. 2008;77:229-57.

7. Huang B, Johansson MJ, Bystrom AS. An early step in wobble uridine tRNA modification requires the Elongator complex. RNA. 2005;11(4):424-36.

8. Otero G, Fellows J, Li Y, de Bizemont T, Dirac AM, Gustafsson CM, et al. Elongator, a multisubunit component of a novel RNA polymerase II holoenzyme for transcriptional elongation. Mol Cell. 1999;3(1):109-18.

9. Slaugenhaupt SA, Blumenfeld A, Gill SP, Leyne M, Mull J, Cuajungco MP, et al. Tissue-specific expression of a splicing mutation in the IKBKAP gene causes familial dysautonomia. Am J Hum Genet. 2001;68(3):598-605.

10. Anderson SL, Coli R, Daly IW, Kichula EA, Rork MJ, Volpi SA, et al. Familial dysautonomia is caused by mutations of the IKAP gene. Am J Hum Genet. 2001;68(3):753-8.

11. Chen YT, Hims MM, Shetty RS, Mull J, Liu L, Leyne M, et al. Loss of mouse Ikbkap, a subunit of elongator, leads to transcriptional deficits and embryonic lethality that can be rescued by human IKBKAP. Mol Cell Biol. 2009:29(3):736-44.

12. George L, Chaverra M, Wolfe L, Thorne J, Close-Davis M, Eibs A, et al. Familial dysautonomia model reveals Ikbkap deletion causes apoptosis of Pax3+ progenitors and peripheral neurons. Proc Natl Acad Sci USA. 2013:110(46):18698-703.

13. Waszak SM, Robinson GW, Gudenas BL, Smith KS, Forget A, Kojic M, et al. Germline Elongator mutations in Sonic Hedgehog medulloblastoma. Nature. 2020;580(7803):396-401

14. Lin FJ, Shen L, Jang CW, Falnes PO, Zhang Y. Ikbkap/Elp1 deficiency causes male infertility by disrupting meiotic progression. PLoS Genet. 2013:9(5):e1003516.

15. Li Q, Fazly AM, Zhou H, Huang S, Zhang Z, Stillman B. The elongator complex interacts with PCNA and modulates transcriptional silencing and sensitivity to DNA damage agents. PLoS Genet. 2009;5(10):e1000684

16. Gunn A, Stark JM. I-Scel-based assays to examine distinct repair outcomes of mammalian chromosomal double strand breaks. Methods Mol Biol. 2012;920:379-91.

17. Fenech $\mathrm{M}$. The in vitro micronucleus technique. Mutat Res. 2000:455(1-2):81-95.
18. Lindberg HK, Wang X, Jarventaus H, Falck GC, Norppa H, Fenech M. Origin of nuclear buds and micronuclei in normal and folate-deprived human lymphocytes. Mutat Res. 2007;617(1-2):33-45.

19. Sancar A, Lindsey-Boltz LA, Unsal-Kacmaz K, Linn S. Molecular mechanisms of mammalian DNA repair and the DNA damage checkpoints. Annu Rev Biochem. 2004;73:39-85.

20. Bekker-Jensen S, Mailand N. Assembly and function of DNA doublestrand break repair foci in mammalian cells. DNA Repair (Amst). 2010:9(12):1219-28.

21. Bonner WM, Redon CE, Dickey JS, Nakamura AJ, Sedelnikova OA, Solier S, et al. GammaH2AX and cancer. Nat Rev Cancer. 2008;8(12):957-67.

22. Moure CM, Gimble FS, Quiocho FA. The crystal structure of the gene targeting homing endonuclease I-Scel reveals the origins of its target site specificity. J Mol Biol. 2003;334(4):685-95.

23. Bennardo N, Cheng A, Huang N, Stark JM. Alternative-NHEJ is a mechanistically distinct pathway of mammalian chromosome break repair. PLoS Genet. 2008;4(6):e1000110.

24. Blackford AN, Jackson SP. ATM, ATR, and DNA-PK: the trinity at the heart of the DNA damage response. Mol Cell. 2017;66(6):801-17.

25. Banin S, Moyal L, Shieh S, Taya Y, Anderson CW, Chessa L, et al. Enhanced phosphorylation of p53 by ATM in response to DNA damage. Science. 1998;281(5383):1674-7.

26. Wold MS. Replication protein A: a heterotrimeric, single-stranded DNAbinding protein required for eukaryotic DNA metabolism. Annu Rev Biochem. 1997:66:61-92.

27. Li $X$, Heyer WD. Homologous recombination in DNA repair and DNA damage tolerance. Cell Res. 2008;18(1):99-113.

28. Sampath P, Pritchard DK, Pabon L, Reinecke H, Schwartz SM, Morris DR, et al. A hierarchical network controls protein translation during murine embryonic stem cell self-renewal and differentiation. Cell Stem Cell. 2008:2(5):448-60

29. Davies AA, Masson JY, Mcllwraith MJ, Stasiak AZ, Stasiak A, Venkitaraman $A R$, et al. Role of BRCA2 in control of the RAD51 recombination and DNA repair protein. Mol Cell. 2001;7(2):273-82.

30. Zhao W, Wiese C, Kwon Y, Hromas R, Sung P. The BRCA tumor suppressor network in chromosome damage repair by homologous recombination. Annu Rev Biochem. 2019:88:221-45

31. Li Z, Otevrel T, Gao Y, Cheng HL, Seed B, Stamato TD, et al. The XRCC4 gene encodes a novel protein involved in DNA double-strand break repair and V(D)J recombination. Cell. 1995:83(7):1079-89.

32. Tsuzuki T, Fujii Y, Sakumi K, Tominaga Y, Nakao K, Sekiguchi M, et al. Targeted disruption of the Rad51 gene leads to lethality in embryonic mice. Proc Natl Acad Sci USA. 1996;93(13):6236-40.

33. Sonoda E, Sasaki MS, Buerstedde JM, Bezzubova O, Shinohara A, Ogawa $\mathrm{H}$, et al. Rad51-deficient vertebrate cells accumulate chromosomal breaks prior to cell death. EMBO J. 1998;17(2):598-608.

34. Klein HL. The consequences of Rad51 overexpression for normal and tumor cells. DNA Repair (Amst). 2008;7(5):686-93.

35. Chen C, Tuck S, Bystrom AS. Defects in tRNA modification associated with neurological and developmental dysfunctions in Caenorhabditis elegans elongator mutants. PLoS Genet. 2009;5(7):e1000561.

36. Kirino Y, Yasukawa T, Ohta S, Akira S, Ishihara K, Watanabe K, et al. Codonspecific translational defect caused by a wobble modification deficiency in mutant tRNA from a human mitochondrial disease. Proc Natl Acad Sci USA. 2004;101(42):15070-5.

37. Yarian C, Marszalek M, Sochacka E, Malkiewicz A, Guenther R, Miskiewicz A, et al. Modified nucleoside dependent Watson-Crick and wobble codon binding by tRNALysUUU species. Biochemistry. 2000:39(44):13390-5.

38. Goffena J, Lefcort F, Zhang Y, Lehrmann E, Chaverra M, Felig J, et al. Elongator and codon bias regulate protein levels in mammalian peripheral neurons. Nat Commun. 2018;9(1):889.

39. Bauer F, Matsuyama A, Candiracci J, Dieu M, Scheliga J, Wolf DA et al. Translational control of cell division by Elongator. Cell Rep. 2012:1(5):424-33.

40. Fernandez-Vazquez J, Vargas-Perez I, Sanso M, Buhne K, Carmona M, Paulo $E$, et al. Modification of tRNA(Lys) UUU by elongator is essential for efficient translation of stress mRNAs. PLoS Genet. 2013;9(7):e1003647.

41. Bauer F, Hermand D. A coordinated codon-dependent regulation of translation by Elongator. Cell Cycle. 2012;11(24):4524-9.

42. Zinshteyn B, Gilbert WV. Loss of a conserved tRNA anticodon modification perturbs cellular signaling. PLoS Genet. 2013;9(8):e1003675. 
43. Hine CM, Li H, Xie L, Mao Z, Seluanov A, Gorbunova V. Regulation of Rad51 promoter. Cell Cycle. 2014;13(13):2038-45.

44. Iwanaga R, Komori H, Ohtani K. Differential regulation of expression of the mammalian DNA repair genes by growth stimulation. Oncogene. 2004;23(53):8581-90.

45. Linke SP, Sengupta S, Khabie N, Jeffries BA, Buchhop S, Miska S, et al. p53 interacts with hRAD51 and hRAD54, and directly modulates homologous recombination. Cancer Res. 2003;63(10):2596-605.

46. Bindra RS, Schaffer PJ, Meng A, Woo J, Maseide K, Roth ME, et al. Downregulation of Rad51 and decreased homologous recombination in hypoxic cancer cells. Mol Cell Biol. 2004;24(19):8504-18.

47. Rodnina MV. The ribosome in action: tuning of translational efficiency and protein folding. Protein Sci. 2016;25(8):1390-406.

48. Shen J, Peng Y, Wei L, Zhang W, Yang L, Lan L, et al. ARID1A deficiency impairs the DNA damage checkpoint and sensitizes cells to PARP inhibitors. Cancer Discov. 2015;5(7):752-67.

49. Brown KK, Montaser-Kouhsari L, Beck AH, Toker A. MERIT40 is an Akt substrate that promotes resolution of DNA damage induced by chemotherapy. Cell Rep. 2015;11(9):1358-66.
50. Okamoto K, Bartocci C, Ouzounov I, Diedrich JK, Yates JR 3rd, Denchi EL. A two-step mechanism for TRF2-mediated chromosome-end protection. Nature. 2013:494(7438):502-5.

51. Zhang F, Ma J, Wu J, Ye L, Cai H, Xia B, et al. PALB2 links BRCA1 and BRCA2 in the DNA-damage response. Curr Biol. 2009;19(6):524-9.

52. Mendoza-Santiesteban CE, Hedges lii TR, Norcliffe-Kaufmann L, Axelrod F, Kaufmann H. Selective retinal ganglion cell loss in familial dysautonomia. J Neurol. 2014;261(4):702-9.

53. Li L, Gruner K, Tourtellotte WG. Retrograde nerve growth factor signaling abnormalities in familial dysautonomia. J Clin Invest. 2020;130(5):2478-87.

\section{Publisher's Note}

Springer Nature remains neutral with regard to jurisdictional claims in published maps and institutional affiliations.
Ready to submit your research? Choose BMC and benefit from:

- fast, convenient online submission

- thorough peer review by experienced researchers in your field

- rapid publication on acceptance

- support for research data, including large and complex data types

- gold Open Access which fosters wider collaboration and increased citations

- maximum visibility for your research: over 100M website views per year

At BMC, research is always in progress.

Learn more biomedcentral.com/submissions 